\title{
3 Pressing Stand and Methods of Processing Measured Data
}

This chapter of the monograph is dedicated to experimental research of densification process in laboratory conditions. This chapter will focus on information summarised in the second chapter, including the process of compaction, the parameters affecting the process, material properties, and so on. Included are the design of an experimental pressing stand, machines, tools, and equipment. The design of a multifactor experiment for verifying the effect of analyzed parameters on the quality of the briquette is also discussed. Data from experiments are evaluated with the aid of several statistical procedures and methods, as well as the design of a mathematical model for compaction, which describes the effect of analyzed parameters and their interactions. In the final part of this chapter, the importance of these experiments is discussed. Additionally, the optimization process for the pressing chamber and the design of software for the optimisation of structural parameters for the pressing chamber are explained. Finally, prediction of the quality of the mould from setup parameters is discussed. This software can provide valuable information for biofuel producers and pressing machine designers.

\subsection{Pressing Stand Design}

The experiment requires the availability of appropriate equipment. Because the necessary experimental equipment was not directly available, I designed it. The design was kept simple, but it was ensured that the finished equipment could meet experimental requirements. First, it was necessary to solve the question of the administration of pressing force. The WPM hydraulic press (Fig. 3.1), which is available in the Institute of Applied Mechanics and Mechatronics, was employed for this design. On the basis of the workplace for this hydraulic press, we measured the maximum limits for the proposed pressing stand construction. Because the hydraulic press WPM is mounted vertically, the stand was adjusted. The hydraulic drive of the press enables the pressing force magnitude and pressing speed to be regulated. This press also provides the operator a simple configuration and readings for force. The maximum possible force applied by the press is $100 \mathrm{kN}$.

The pressing stand construction, which enables the measurement of several parameters, was also considered. Because the material's water content and fraction size are so-called material properties, they were not considered in the first phase of the experiment. However, it was thus necessary to design a construction that would enable the pressing temperature and other design parameters to be easily changed. 
This was not the subject of our research, but for future purposes, it was considered for the institute to investigate these parameters. Fig 3.2 shows a 3D model of the pressing stand, and the constructed stand itself can be seen in Fig 3.3. The designed pressing stand allows for the study of the length of the pressing chamber, the effect of the conicity of the chamber walls, and the change of friction factor between the briquette material and the chamber to be investigated. After minor adjustments, it is also possible to study the effect of diameter change of the chamber.

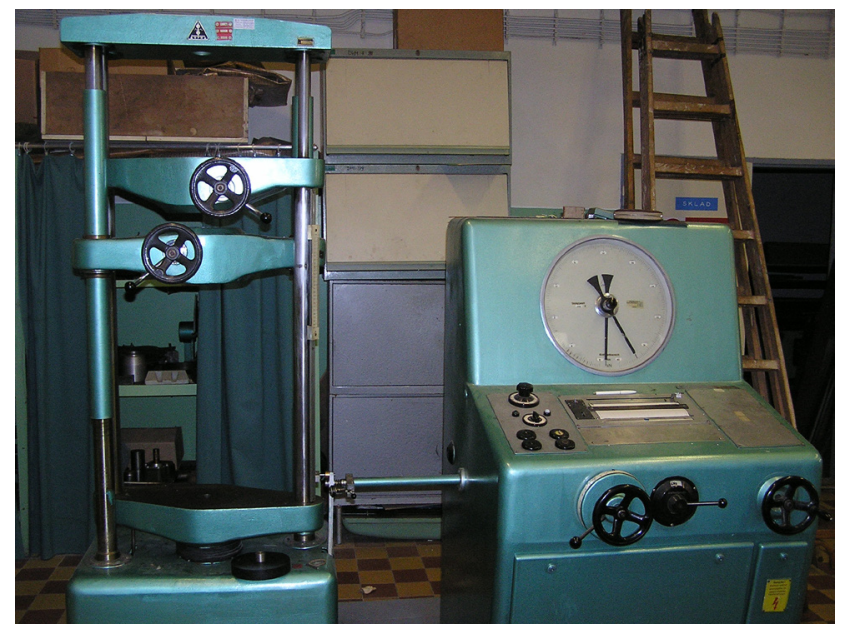

Figure 3.1: Hydraulic press used in the experiment.

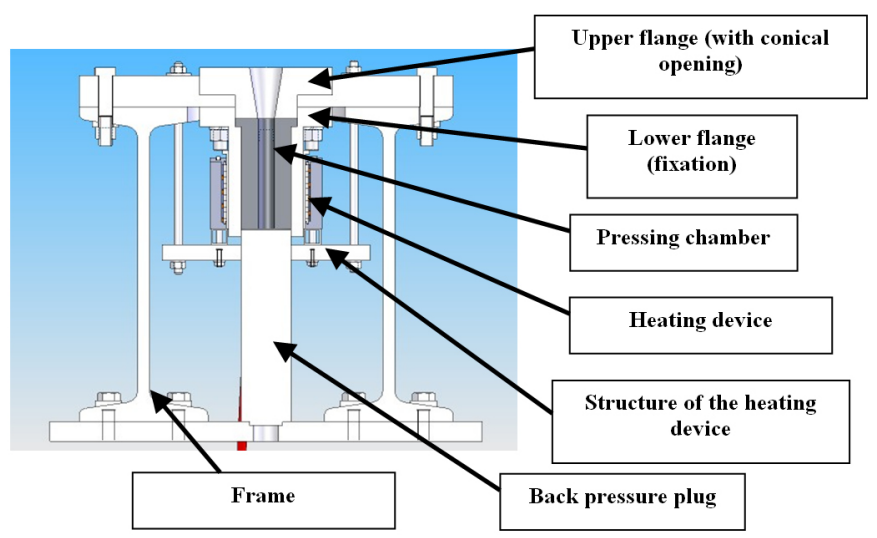

Figure 3.2: 3D section of the press stand and its basic components (Križan, 2009). 


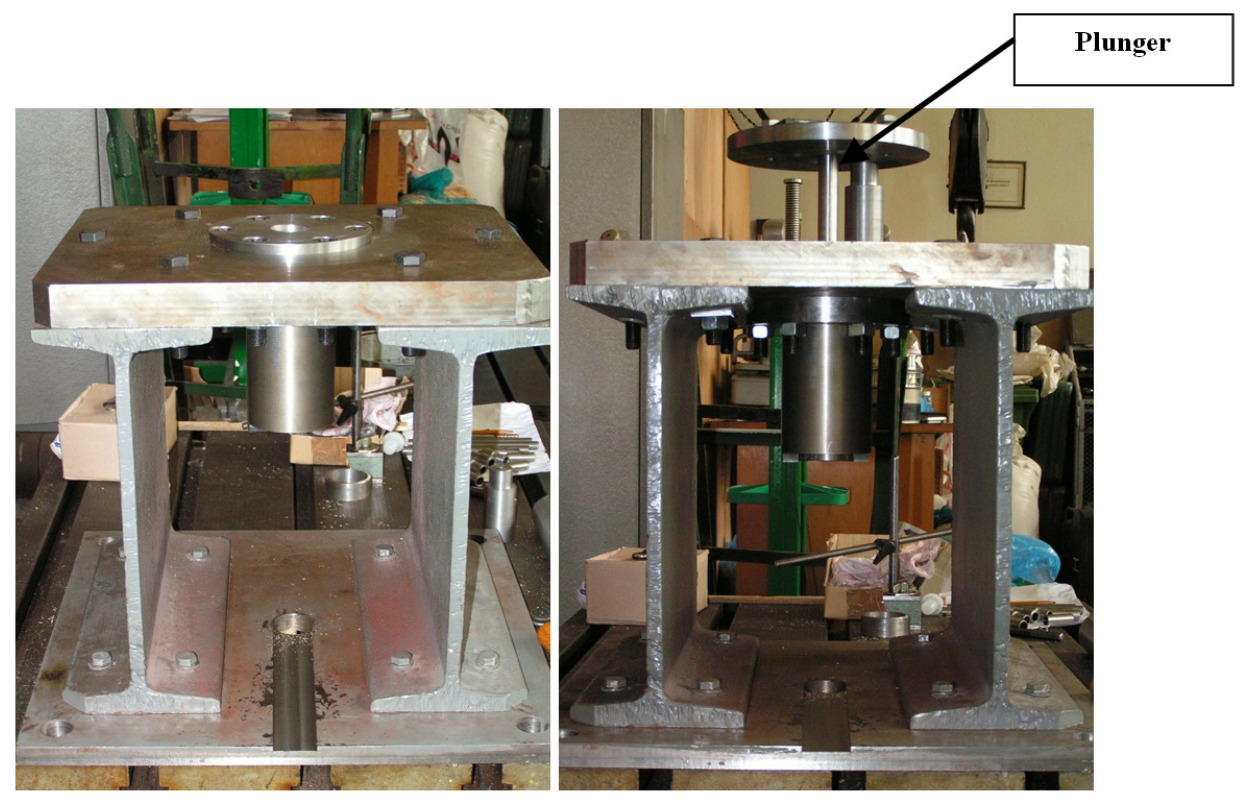

Figure 3.3: Constructed pressing stand before installing into the hydraulic press (Križan, 2009).

The next step was the design of a heating device for the pressing stand. With a continuous mode of compaction, temperature fluctuations arise as a result of the machine kinematics and friction between the pressing tool and material. But, in our case, this is excluded. The heating device provides heat to the chamber and thus heats the workspace while running the process. An additional requirement from the heating device was the knowledge and control of the temperature inside the chamber and a flexible response when changing this internal temperature. From the previous analysis, we know that the temperature inside the chamber during the pressing process affects the briquette. After finding an appropriate heating device manufacturer, three temperature sensors were installed into the chamber evenly along the length of the pressing opening, as can be seen in Fig. 3.4. Obviously, the preliminarily calculation of heat transfer was also investigated. A heating coil, which is generally used in chemical laboratories while heating solvents in flasks, was employed as the heating element. The coil's power is $600 \mathrm{~W}$, and the coil was fixed to the bottom flange of the stand (Fig. 3.5). The heating coil is controlled by another heat sensor, which was fixed into the opening diagonally across the chamber, $2 \mathrm{~mm}$ under the edge of the pressing opening (Fig. 3.4b and 3.5a). This setup provided the heating object used for the experiments. 


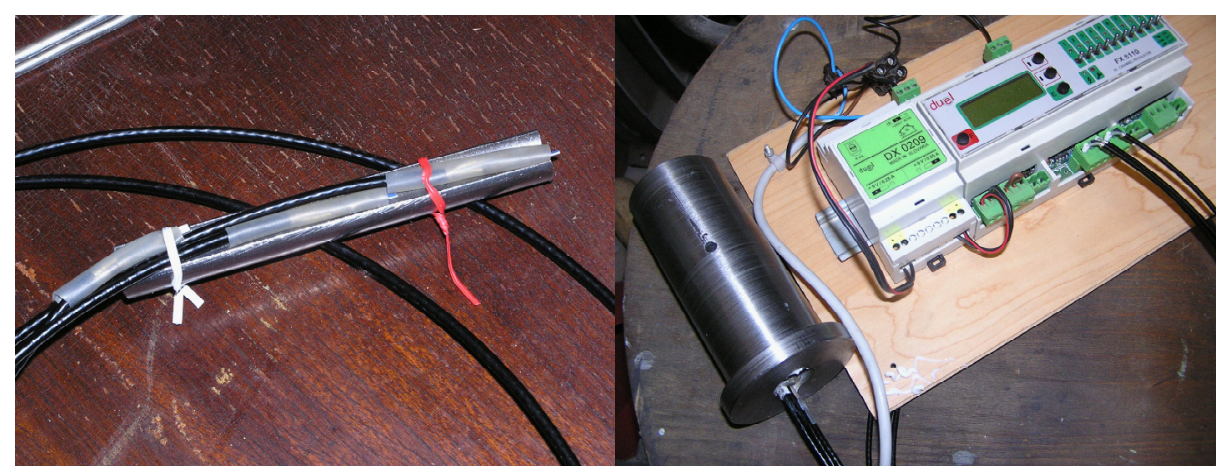

a.)

b.)

Figure 3.4: Test and verification of heat transfer- a.) sensor placement; b.) pressing chamber with mounted sensors (Križan, 2009).

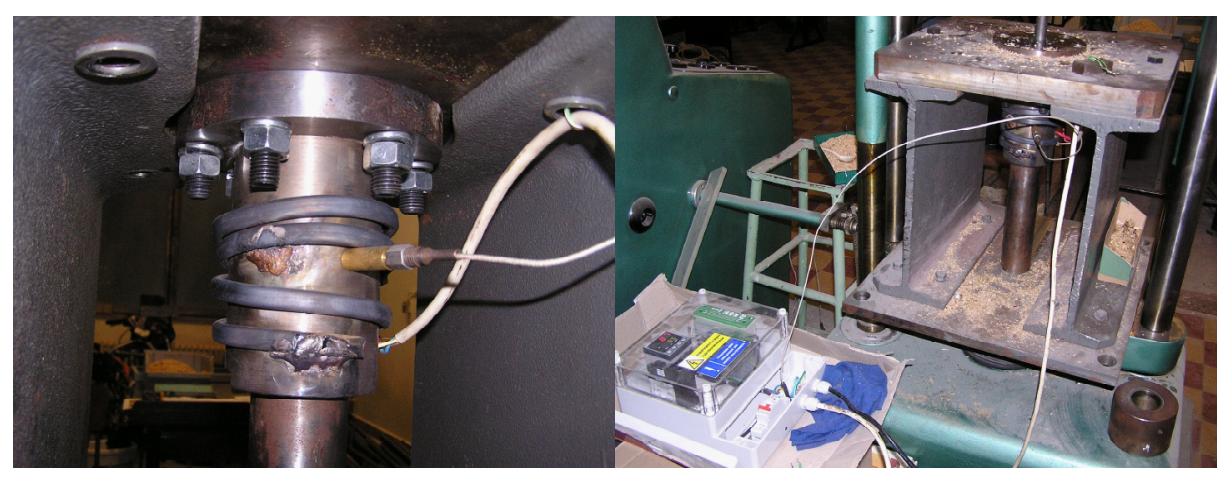

a.)

b.)

Figure 3.5: Fixed heating coil- a.) mounted heat sensor; b.) connection of heating coil and sensors to the control unit (Križan, 2009).

Based on the results from a preliminary heat transfer test, a heating device with an intelligent heating coil was designed. It allowed for the smooth balance of heat fluctuations with high sensitivity to maintain the temperature around the nominal pressing temperature. This heating device supplies the chamber with heat according to a configured value within a few minutes. After constructing and mounting the heating system, the pressing stand was ready to meet the requirements of the experiment. 


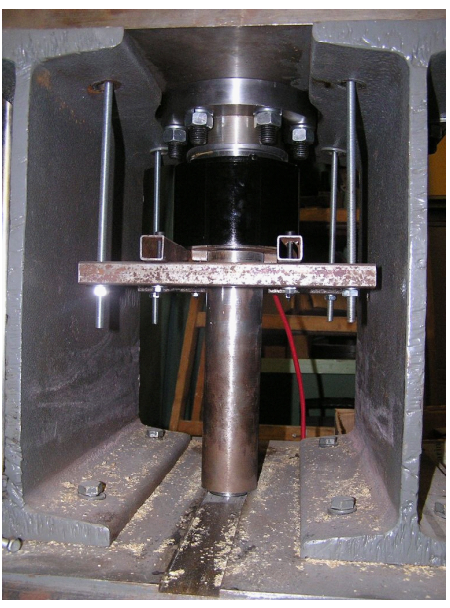

a.)

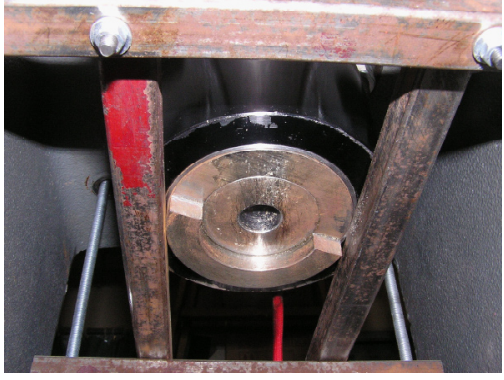

b.)

Figure 3.6: Designed heating device - a.) mounted heating device; b.) bottom view (Križan, 2009).
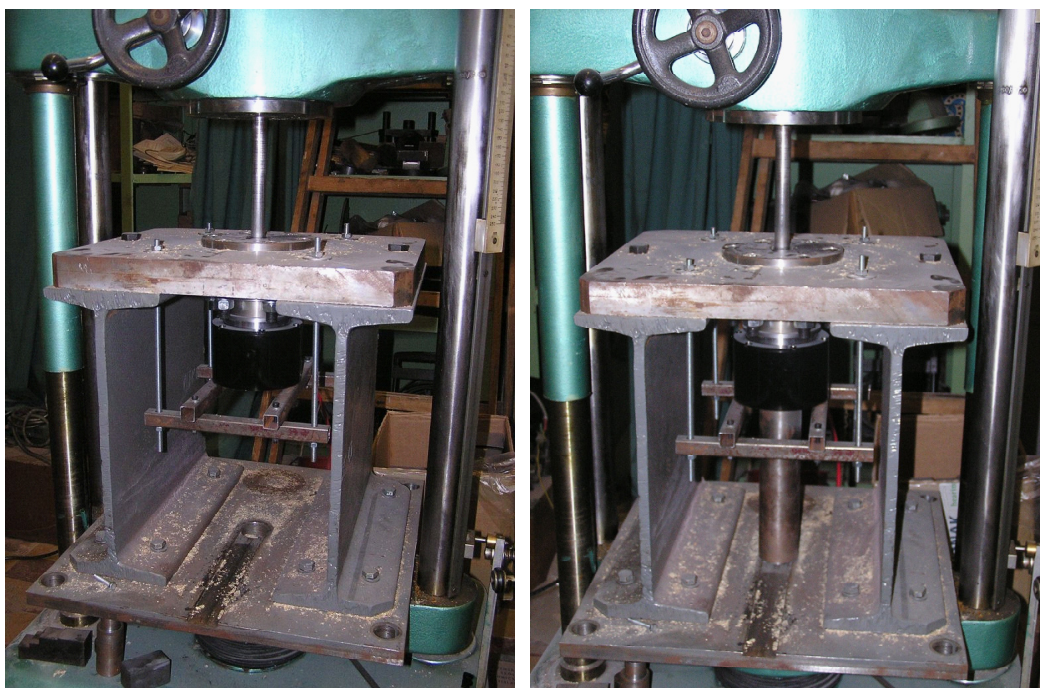

Figure 3.7: Experimental pressing stand prepared for the experiment (Križan, 2009). 


\subsection{Experiment Design and Description}

It is necessary to design a set of experiments to verify the effects of analyzed parameters so that a mathematical model for compaction can be constructed. The model was aimed to incorporate four basic parameters and their mutual interactions affecting the resulting briquette. On the basis of the previous analysis, we recognize that it is necessary to design a multifactor experiment to study the effect of pressing pressure, temperature, size of entering fraction, and moisture content of the material. In this part of the chapter, we will describe the design and procedure for the experiments.

\subsubsection{Experiment Design}

If we consider a system with four parameters, it is possible to assume the input value as a function with four variables $\rho=f\left(p, T, w_{r}, L\right)$. Based on this concept and advice and recommendations given by experts in this area, the experimental procedure according to a centered composite design was designed.

Table 3.1: Centered composite design for the experiment requirements (Chudý \& et al., 1999).

\begin{tabular}{|l|l|l|l|l|}
\hline $\mathbf{i}$ & $\mathbf{k} \mathbf{1}$ & $\mathbf{k} 2$ & $\mathbf{k} 3$ & $\mathbf{k 4}$ \\
\hline 1 & -1 & -1 & -1 & -1 \\
\hline 2 & 1 & -1 & -1 & -1 \\
\hline 3 & -1 & 1 & -1 & 1 \\
\hline 4 & 1 & 1 & -1 & 1 \\
\hline 5 & -1 & -1 & 1 & -1 \\
\hline 6 & 1 & -1 & 1 & -1 \\
\hline 7 & -1 & 1 & 1 & 1 \\
\hline 8 & 1 & 1 & 1 & 1 \\
\hline 9 & -1 & -1 & -1 & 1 \\
\hline 10 & 1 & -1 & -1 & 1 \\
\hline 11 & -1 & 1 & -1 & -1 \\
\hline 12 & 1 & 1 & -1 & -1 \\
\hline 13 & -1 & -1 & 1 & 1 \\
\hline 14 & 1 & -1 & 1 & 1 \\
\hline 15 & -1 & 1 & 1 & -1 \\
\hline 16 & 1 & 1 & 1 & -1 \\
\hline
\end{tabular}

\begin{tabular}{lllll}
\hline $\mathbf{i}$ & $\mathbf{k 1}$ & $\mathbf{k 2}$ & $\mathbf{k 3}$ & $\mathbf{k 4}$ \\
\hline 17 & $\alpha-$ & 0 & 0 & 0 \\
\hline 18 & $\alpha$ & 0 & 0 & 0 \\
\hline 19 & 0 & $\alpha-$ & 0 & 0 \\
\hline 20 & 0 & $\alpha$ & 0 & 0 \\
\hline 21 & 0 & 0 & $\alpha-$ & 0 \\
\hline 22 & 0 & 0 & $\alpha$ & 0 \\
\hline 23 & 0 & 0 & 0 & $\alpha-$ \\
\hline 24 & 0 & 0 & 0 & $\alpha$ \\
\hline 25 & 0 & 0 & 0 & 0 \\
\hline
\end{tabular}

- design core

- star points

- central points

i - number of configurations

$\mathrm{k}_{1}$ - pressing pressure

$\mathrm{k}_{2}$ - pressing temperature

$\mathrm{k}_{3}$ - material moisture content

$\mathrm{k}_{4}$ - size of entering fraction 
These designs belong to the most commonly used composite designs (Horníková, 2009). They consist of three parts:

1. Core of the design - composed of a total factorial experiment of $2^{k}(\mathrm{k}=$ number of examined factors). If $k \leq 4$, it is necessary to use as a core the total experiment $2^{k}$. In our case, $2^{4}=16$.

2. Star points - points that lie on the coordinate axes with a distance of $\alpha>0$ from the center of the design. Their quantity is $2 k$. In our case, it is $2 k=2.4=8$.

3. Central points - points in the center of design. In our case, we would suggest one central point

An appropriate selection of $\alpha$ and the number of central points can affect the properties of the designs.

Table 3.2: Set parameter levels (Križan \& Svátek, 2007; 2008).

\begin{tabular}{lllllll}
\hline factor & unit & $-\boldsymbol{\alpha}$ & $\mathbf{- 1}$ & $\mathbf{0}$ & $\mathbf{1}$ & $\boldsymbol{\alpha}$ \\
\hline pressure & $(\mathrm{MPa})$ & 63 & 95 & 127 & 159 & 191 \\
temperature & $(\underline{\mathrm{o}} \mathrm{C})$ & 55 & 85 & 100 & 115 & 130 \\
humidity & $(\%)$ & 5 & 8 & 10 & 12 & 15 \\
size & $(\mathrm{mm})$ & 0.5 & 1 & 2 & 4 & $4<$ \\
\hline
\end{tabular}

For specific and exact evaluation of the measured data, it was necessary to set the values for each examined parameter. The levels were defined with respect to compaction and the pressing stand. The experiment was performed with a total factorial of $2^{4}$ (Wimmer \& et al., 2001) according to Tab. 3.1 (orange fields-design core). To obtain the most precise experiment, the interval of validity is constricted. From the originally set levels of examined parameters (Tab. 3.2), the experiments on levels listed in Tab. 3.3 (where specific values of examined parameters are listed) were set.

\subsubsection{Pressing Procedure and Apparatus Used}

The aim of the experiments was to observe the quality of the briquette in relation to the examined factors (pressing pressure, pressing temperature, material moisture content, and the size of entering fraction). The quality of the briquette was evaluated according to resulting density. Due to the complexity of the experiments, only one raw material, pine sawdust, was considered. The experiments were carried out on the constructed pressing stand. At each set level, according to the centered composite 
Table 3.3: Centered composite design with specific value of examined parameters (Križan \& Svátek, 2007; 2008).

\begin{tabular}{lllll}
\hline $\mathbf{i}$ & $\mathbf{p}(\mathbf{M P a})$ & $\mathbf{T}\left({ }^{\mathbf{o}} \mathbf{C}\right)$ & $\mathbf{w}(\%)$ & $\mathbf{L}(\mathbf{m m})$ \\
\hline 1 & 95 & 85 & 8 & 1 \\
2 & 159 & 85 & 8 & 1 \\
3 & 95 & 115 & 8 & 4 \\
4 & 159 & 115 & 8 & 4 \\
5 & 95 & 85 & 12 & 1 \\
6 & 159 & 85 & 12 & 1 \\
7 & 95 & 115 & 12 & 4 \\
8 & 159 & 115 & 12 & 4 \\
9 & 95 & 85 & 8 & 4 \\
10 & 159 & 85 & 8 & 4 \\
11 & 95 & 115 & 8 & 1 \\
12 & 159 & 115 & 8 & 1 \\
13 & 95 & 85 & 12 & 4 \\
14 & 159 & 85 & 12 & 4 \\
15 & 95 & 115 & 12 & 1 \\
16 & 159 & 115 & 12 & 1 \\
\hline
\end{tabular}

design, seven briquettes were made. For the purpose of further evaluation of the measured data (analysis of diameters, variance analysis, effect method, etc.), the literature indicates a minimum of six values to be obtained at each configuration level. The experimental procedure for compaction was as follows:

\section{Material preparation}

Because in the experimental plan there exist several levels of size and water content, a sieving analysis was conducted to ensure various values of fraction and moisture. For the sieving analysis, a sieving apparatus with different sizes of openings $0.5,1,2$, and $4 \mathrm{~mm}$ (Fig. 3.8) was employed. 


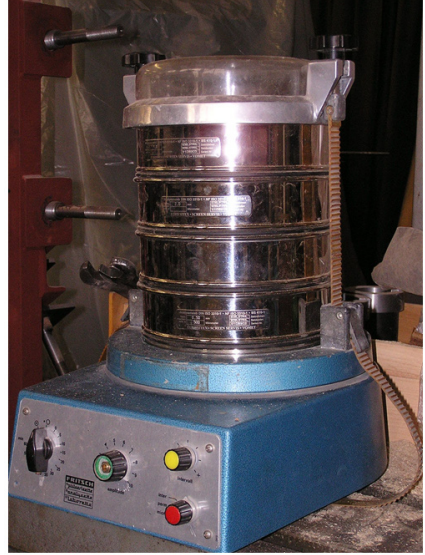

a.)

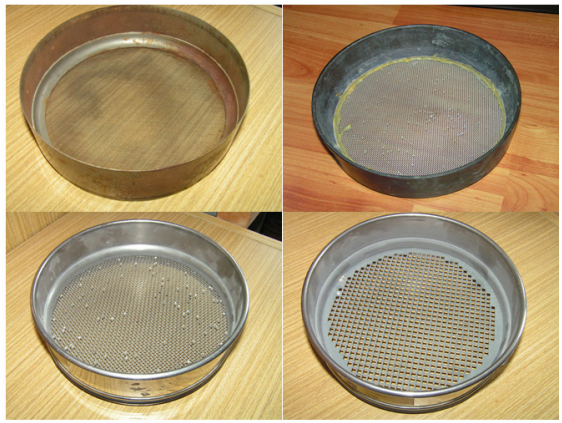

b.)

Figure 3.8: Tools used in sieving analysis-a.) sieving apparatus; b.) laboratory sieves.

Different moisture content values in the sawdust were obtained by drying in the laboratory (Fig. 3.9) and controlling specific values with the aid of a digital humidity meter GMH 3830 (Fig. 3.10).

\section{Dosage and filling of the pressing chamber}

Because the volume of the opening in the pressing chamber and the ratio of compaction are known, the amount of sawdust needed to fill the opening can be determined. This is how filling the opening with the same dosage of weighed sawdust was done. The pressing chamber is of cylindrical shape with a diameter of $20 \mathrm{~mm}$. The principle of pressing is vertical; therefore, when filling the chamber, it is important to use a backpressure plug, which prevents free sawdust from exiting the chamber during pressing.

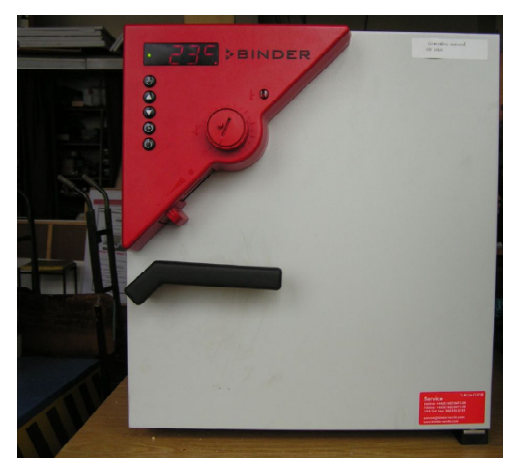

Figure 3.9: Laboratory dryer BINDER ED 23. 


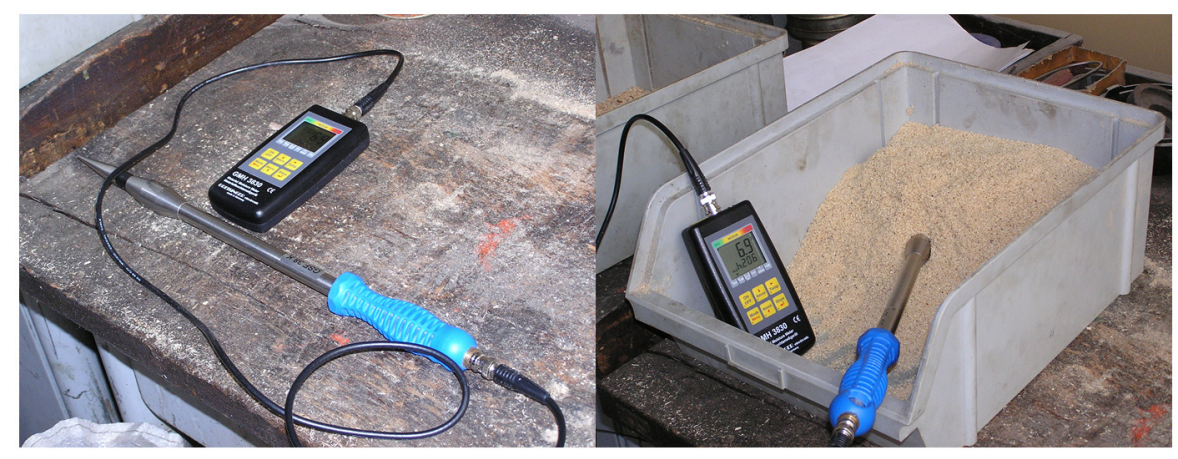

a.)

b.)

Figure 3.10: Digital humidity meter GMH 3830 with GSF $38 \mathrm{~K}$ probe for granular materials-a.) digital humidity meter; b.) measuring humidity.

\section{Pressing procedure}

The pressing process can begin when the material, which is prepared prior to loading according to the desired fraction size and moisture content, is inside the pressing chamber. During pressing, the experimental plan and configuration of the defined value levels of the examined parameters are closely followed. The control panel of the heating apparatus must indicate that the temperature inside the chamber is equal to the set temperature before filling the pressing opening with sawdust can begin(Fig. 3.11a.). Heating the pressing chamber is used to facilitate the plasticisation of lignin in the material's cellular structure, and mainly because it enables the needed decrease of pressure for obtaining the required quality of the briquette. After filling the pressing opening with sawdust, a pressing pressure according to the experimental plan is set (Fig. 3.11b.). After releasing of the pressure, the pressing opening is filled to the edges (Fig. 3.11c.). Subsequently, the sawdust is pressed into a compact form with a set pressing force according to the experimental plan (Fig. 3.11d.). After releasing the pressure, the backpressure plug is released, and the briquette is pushed outside of the pressing chamber (Fig. 3.11e.). In this way, one briquette is made at a time; for each configuration, according to the centered composite design, at least six briquettes for each setting has to be made. In order to fulfil this condition, seven briquettes per configuration were made.

\section{Briquette dimensions, weight, and stability}

After pressing the briquette, its diameter and length are measured. Using two separate callipers for each dimension prevents correlation of measured values, which would appear while calculating inaccuracies of the measurement. With the aid of a digital scale, the briquette is weighed (Fig. 3.12). The briquette is allowed to sit for 

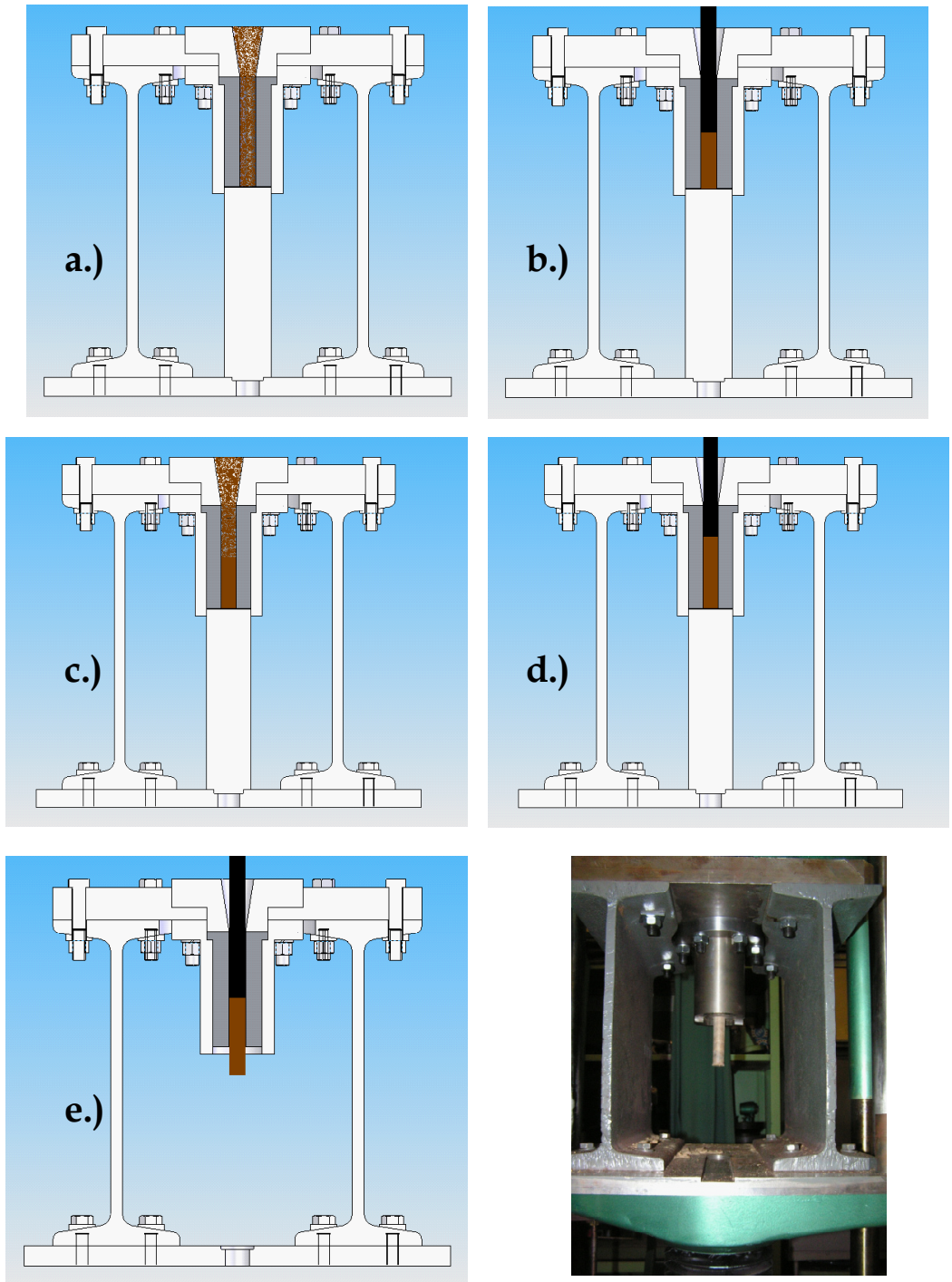

Figure 3.11: Pressing phases of wooden sawdust

a defined length of time to dilate and stabilize. After the stabilization period, the diameter, length, and weight of the briquette is measured again. These values are the foundation for determining the density according to DIN 52182. Densities obtained at one configuration according to the experimental plan are averaged and are used when evaluating the measured data. 
The described procedure was repeated according to the designed experimental plan and centered composite design.

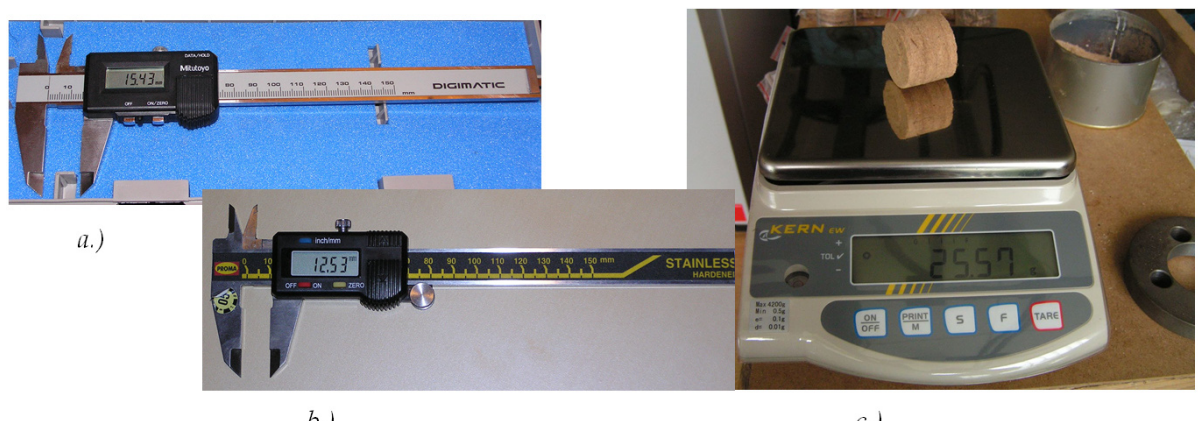

b.)

c.)

Figure 3.12: Used instruments- a.) digital caliper 1; b.) digital caliper 2; c.) digital scale KERN.

\subsection{Processing Methods and Experiment Evaluation}

With the procedure described in previous section, estimates were obtained for the measured and calculated values of density, which are listed in Tab. 3.4. In the left part of the table, the values of the parameters at which the experiments were performed according to the centered composite design are given. In Tab. 3.4, the values of measured parameters can be seen as $\rho_{1}, \rho_{2}, \ldots ., \rho_{j}$, where $j=1,2, \ldots, r$ (in our case, $r=7$ ), for experiments 1 to 16 . When evaluating, only measured values which characterize the core of the experiment for the centered composite design $2^{4}=16$ are used (Tab.3.1). From these measured values, the selection averages are calculated (Chudý \& et al., 1999; Palenčár \& Jankura, 2005):

$$
\overline{\rho_{j}}=\frac{\sum_{j=1}^{r} \rho_{j}}{r}
$$

for $j=1,2, \ldots, r$ (in our case, $r=7$ ).

These selection averages are also listed in Tab. 3.4 and are the basis for experimental evaluation.

In this chapter, the processing methods and evaluation of the experiment are described. Initial information about the effect of a particular factor is obtained 


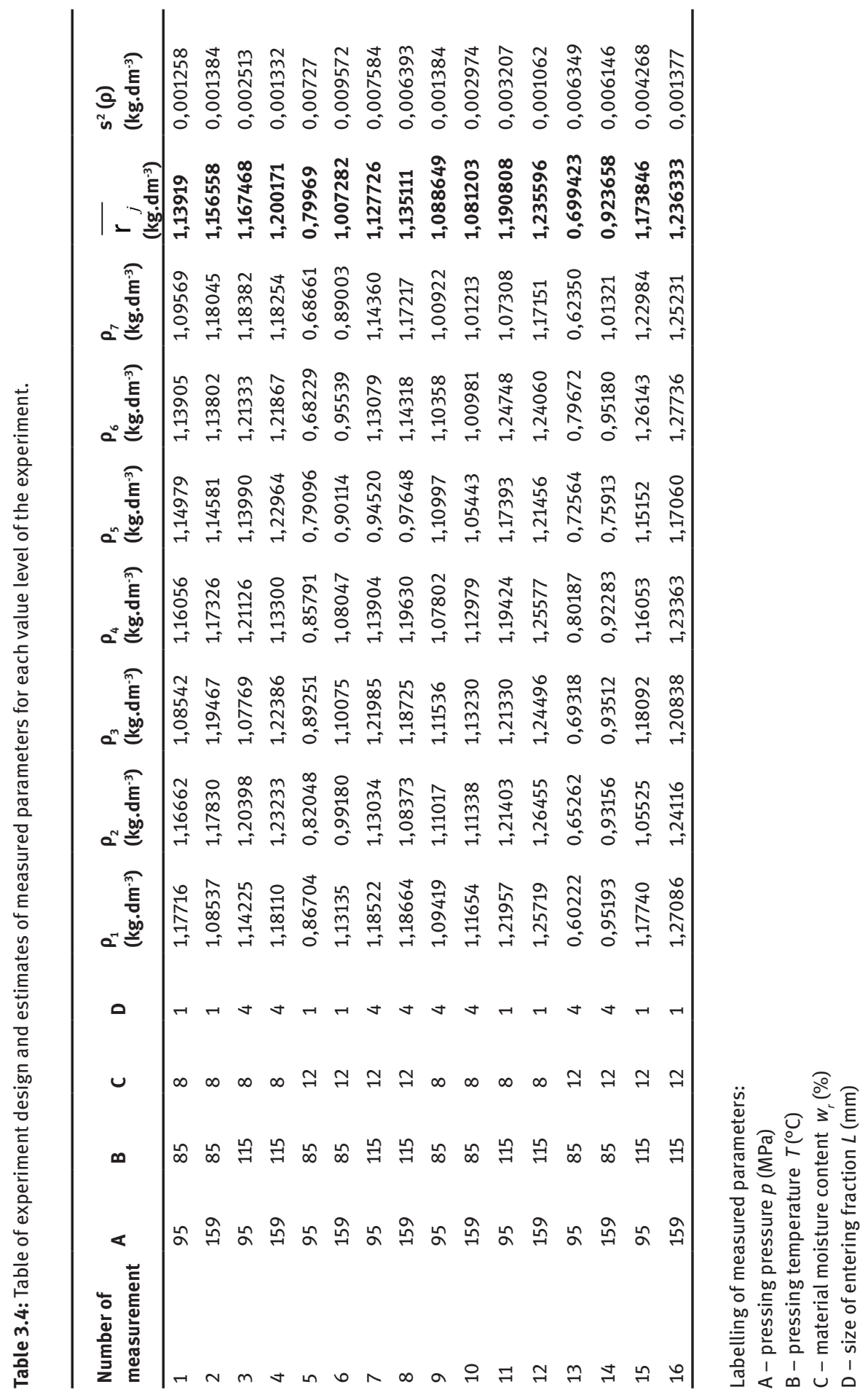


mostly with the aid of the multivariation diagram. In a multivariation diagram, the maximum and minimum values of a certain group are shown. The variability in one group is characterized by a certain range that corresponds to the length of the segment connecting both points. This diagram allows us to monitor level changes and variability at the same time. The multivariation diagram for our experiments is illustrated in Fig. 3.13.

When the multivariation diagram indicates differences in averages and variability on several levels, it is possible to use statistical tests for further verification. Evaluating the divergence of averages in groups makes it possible to use procedures derived from the control chart - average analysis (ANOM) or variance analysis (ANOVA). Both these methods assume normal distribution of the observed character and the same variability for some groups, which indicates an insignificant difference between selection distribution and range. The second of these assumptions verifies whether the Bartlett's test, typically used in relation with ANOVA or by the control diagram of range (R-diagram) or for variance ( $\mathrm{s}^{2}$-diagram), correspond by their character to ANOM.

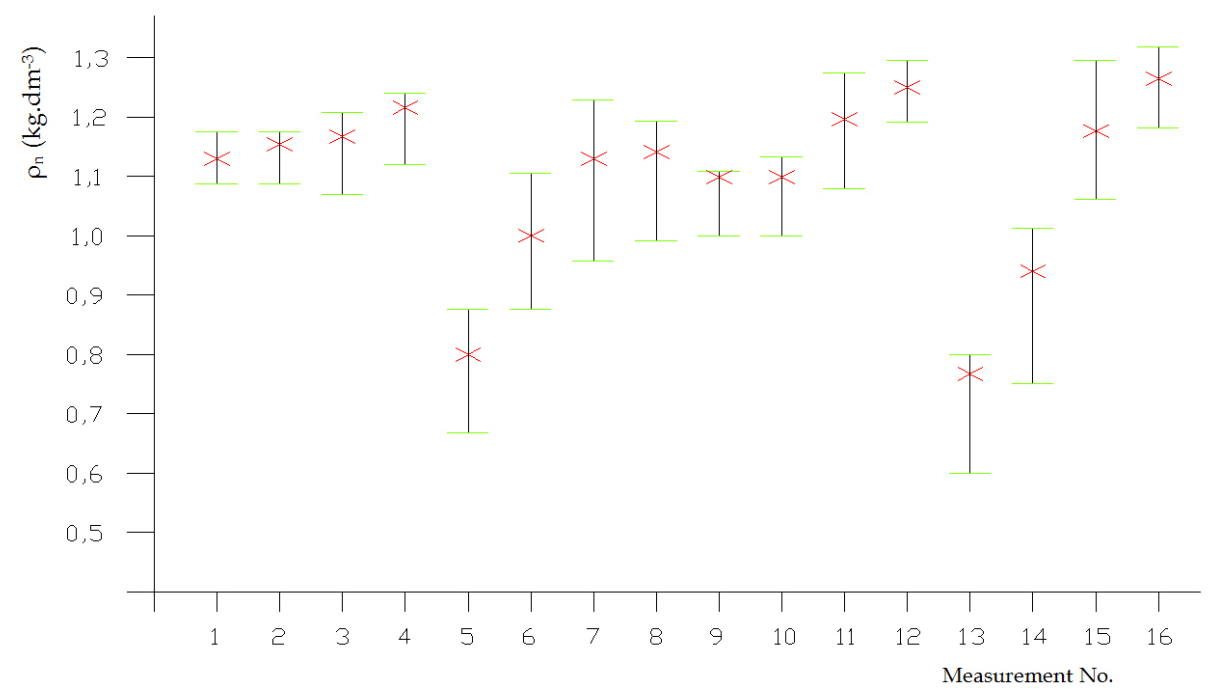

Figure 3.13: Multivariation diagram.

\subsubsection{Average Analysis and Variance Equality Test Using Bartlett's Test}

The first step in evaluation is to perform an average analysis and test the variance equality hypothesis using Bartlett’s test (Palenčár \& et al., 2001). This test can be employed when differences between selection variances $s_{i}^{2}$ or 
the range $R_{i}$ are insignificant. This test is based on $k$ independent selections $y_{11}, y_{12}, \ldots y_{1 n}, y_{21}, y_{22}, \ldots, y_{k 1}, y_{k 2}, \ldots, y_{k n k}$, where $n_{i}>6 ; i=1,2 \ldots, k$.

Represented by:

$$
s_{i}^{2}=\frac{1}{n_{i}-1} \cdot\left(\sum_{j=1}^{n_{j}} y_{i j}^{2}-n_{i} \bar{y}_{i}^{2}\right)
$$

Where:

$$
\begin{gathered}
\bar{y}_{i}=\frac{1}{n_{j}} \sum_{j=1}^{n_{j}} y_{i j}, \mathrm{i}=1,2, \ldots ., \mathrm{k} \\
s^{2}=\frac{1}{n-k} \sum_{i=1}^{k}\left(n_{i}-1\right) \cdot s_{i}^{2} \\
C=1+\frac{1}{3 .(k-1)}\left(\sum_{i=1}^{k} \frac{1}{n_{i}-1}-\frac{1}{n-k}\right)
\end{gathered}
$$

and $n=\sum_{i=1}^{k} n_{i}$

In the following steps, the variance equality hypothesis is tested $H_{0}: \sigma_{1}^{2}=\sigma_{2}^{2}=\ldots \ldots \ldots \ldots \ldots=\sigma_{k}^{2}$ by means of Bartlett's testing statistic:

$$
B=\frac{1}{C} \cdot\left[(n-k) \ln s^{2}-\sum_{i=1}^{k}\left(n_{i}-1\right) \ln s_{i}^{2}\right]
$$

Hypothesis $H_{0}$ is rejected at a significance level of $\alpha$, if: $B \geq \chi^{2}(k-1, \alpha)$ resp. $B \geq \chi_{1-\alpha}^{2}(k-1)$

When using this test, it is necessary that the selection ranges are sufficiently large. In the relevant literature, a condition is usually mentioned where its validity is $n_{i}>$ 6 for each $i=1,2, \ldots, k$. In this case, 16 measurements with 7 repetitions were made, meaning $n_{i}=7$ for each $i=1,2, \ldots, 16$. For this degree of calculation, with respect to the 
large nature of the data, the program STATGRAPHIC S Plus was used. The results of the analysis are the sigma values for each measurement.

Outputs from STATGRAPHIC S Plus:

\begin{tabular}{|c|c|c|c|}
\hline n.m. & n.values & $\frac{\text { average value }}{\rho_{j}}\left(\mathrm{~kg} \cdot \mathrm{dm}^{-3}\right)$ & $\begin{array}{l}\text { variance } \\
\mathrm{s}^{2}(\rho)\left(\mathrm{kg} \cdot \mathrm{dm}^{-3}\right)\end{array}$ \\
\hline Column_1 & 7 & 1.13919 & 0.00125764 \\
\hline Column_2 & 7 & 1.15656 & 0.00138368 \\
\hline Column_3 & 7 & 1.16747 & 0.00251308 \\
\hline Column_4 & 7 & 1.20017 & 0.001332 \\
\hline Column_5 & 7 & 0.79969 & 0.00727022 \\
\hline Column_6 & 7 & 1.00728 & 0.00957224 \\
\hline Column_7 & 7 & 1.12773 & 0.00758404 \\
\hline Column_8 & 7 & 1.13511 & 0.00639349 \\
\hline Column_9 & 7 & 1.08865 & 0.00138447 \\
\hline Column_10 & 7 & 1.0812 & 0.00297365 \\
\hline Column_11 & 7 & 1.19081 & 0.00320678 \\
\hline Column_12 & 7 & 1.2356 & 0.00106233 \\
\hline Column_13 & 7 & 0.6994 & 0.00634858 \\
\hline Column_14 & 7 & 0.923658 & 0.00614595 \\
\hline Column_15 & 7 & 1.17385 & 0.00426814 \\
\hline Column_16 & 7 & 1.23633 & 0.00137731 \\
\hline & $112^{\star}$ & $1.08517^{\star \star}$ & $0.026123^{\star \star}$ \\
\hline
\end{tabular}

The next step was the evaluation of the hypothesis based on Bartlett's test.

Variance control results - STATGRAPHIC S Plus program output:

Cochran's test: $0.149394 \quad P$ - value $=0.339438$

Bartlett's test: $1.30317 \quad P$ - value $=0.0650303$

Hartley's test: 9.0106

The results of Bartlett's test obtained using the program STATGRAPHIC S Plus is the value $P$ - the level of calculated significance, according to which the hypothesis $H_{0}$ is accepted or rejected on the level of specified significance $\alpha=95 \%$.

This means that, in order to accept the variance equality hypothesis, a value of $P$ larger than 0.05 is necessary (Palenčár \& et al., 2001). The analysis results returned values of $\mathrm{P}=0.55001$, which indicates that the hypothesis is acceptable. In this way, we satisfy one of the conditions for additional variance analysis using the method ANOVA. The selection can be regarded as homogeneous with respect to variability. This fact, based on the results, was also confirmed by the value of Cochran's tests. 


\subsubsection{Experiment Evaluation by the Variance Analysis Method (ANOVA)}

The affecting factors are seen through the significant differences between selection averages (Chudý \& et al., 1999; Palenčár \& et al., 2001). This means that the fluctuation of the selection averages over the total average is larger than the fluctuation of each result within the selection. Variance analysis, contrary to selecting averages, compares two variance estimates describing the variability type. For this analysis, the same assumptions apply for the averaging analysis. The role of the variance analysis is to find the effect of one or more factors on the average value of independent, normally divided, random variables with the same variance.

The test was made in two steps:

- In the first step, the hypothesis $H_{o}: \mu 1=\mu 2=\ldots=\mu k$ is tested on a set level of significance $\alpha$ if $H_{0}$ is rejected.

- $\quad$ The groups that differ from each other are identified.

Table 3.5: Variance analysis table (Chudý \& et al., 1999).

\begin{tabular}{lllll}
\hline Variability source & Sum f squares & $\begin{array}{l}\text { Degree of } \\
\text { freedom }\end{array}$ & Average square & ratio F \\
\hline Factor $A$ & $\mathrm{~S}_{\mathrm{A}}=\{1\}-\{3\}$ & $\mathrm{I}-1$ & $\mathrm{~S}_{\mathrm{A}}{ }^{2}=\mathrm{S}_{\mathrm{A}} /(\mathrm{I}-1)$ & $\mathrm{S}_{\mathrm{A}}{ }^{2} / \mathrm{S}_{\mathrm{e}}{ }^{2}$ \\
residual & $\mathrm{S}_{\mathrm{e}}=\{2\}-\{1\}$ & $\mathrm{n}-1$ & $\mathrm{~S}_{\mathrm{e}}{ }^{2}=\mathrm{S}_{\mathrm{e}} /(\mathrm{n}-1)$ & $\mathrm{S}_{\mathrm{A}}{ }^{2} / \mathrm{S}_{\mathrm{e}}{ }^{2}$ \\
\hline
\end{tabular}

After substituting in the formulas from Table 3.5, specific values can be obtained. In the table for the ANOVA method (F values), for the defined significance $\alpha$, a certain number of degrees of freedom and critical value $F_{1-\alpha}$, are found. In another step, the ratio $S_{A}{ }^{2} / S_{e}{ }^{2}$ is compared with the critical value $F_{1-\alpha}$ from the table. If the value of the ratio $S_{A}{ }^{2} / S_{e}^{2}$ is larger than that of the critical value of $F_{1-\alpha}$, the effects of this factor are considered to be significant (Palenčár \& et al., 2001).

For this analysis, the program STATGRAPHIC $S$ Plus was used once again. The range of examined parameters for the next step is defined as follows:

Table 3.6: Examined parameter range table.

\begin{tabular}{|c|c|c|}
\hline Factor & Unit & Range \\
\hline Pressure & $(\mathrm{MPa})$ & $95-159$ \\
\hline Temperature & $\left({ }^{\circ} \mathrm{C}\right)$ & $85-115$ \\
\hline Moisture content & (\%) & $8-12$ \\
\hline Fraction size & $(\mathrm{mm})$ & $1-4$ \\
\hline
\end{tabular}


The next step was the evaluation process itself and the determination of each factor's significance with the aid of the program STATGRAPHIC S Plus. The analysis results are listed in Table 3.7.

The effect of each parameter or its significance is given by the value of $P$ - level of calculated significance, which we compared. The effect of the parameter is considered important at a significance level of $P^{*}(95 \%)$; when the value of $P$ is less than $\left(1-P^{*}\right)$, this means $P<0$. 05. From this, and based on the analysis results listed in Table 3.7, it is implied that the order of significance for each of the parameters are as follows: pressing temperature, material moisture content, pressing pressure, fraction size.

Table 3.7: Variance analysis table- results (Križan \& Svátek, 2007; 2008).

\begin{tabular}{|c|c|c|c|c|c|c|}
\hline Variability source & $\begin{array}{l}\text { Sum of } \\
\text { squares }\end{array}$ & $\begin{array}{l}\text { Degrees } \\
\text { of } \\
\text { freedom }\end{array}$ & $\begin{array}{l}\text { Average } \\
\text { square }\end{array}$ & $\begin{array}{l}\text { Ratio } \\
\text { F }\end{array}$ & $\begin{array}{l}\text { Value } \\
\text { P }\end{array}$ & significance \\
\hline A - pressure „p“ & 0.0210523 & 1 & 0.0210523 & 3.40 & 0.0923 & $3^{\text {rd }}$ \\
\hline B - temperature „T“ & 0.13857 & 1 & 0.13857 & 22.37 & 0.0006 & $1^{\text {st }}$ \\
\hline C - moisture content „,W “ & 0.0709649 & 1 & 0.0709649 & 11.46 & 0.0061 & $2^{\text {nd }}$ \\
\hline D - fraction size „L“ & 0.0107137 & 1 & 0.0107137 & 1.73 & 0.2152 & $4^{\text {th }}$ \\
\hline Residual & 0.0681389 & 11 & 0.00619445 & & & \\
\hline Sum & 0.30944 & 15 & & & & \\
\hline
\end{tabular}

\subsubsection{Experiment Evaluation with Factor Effect Method}

For a closer determination, not only of the effect of each parameter but also of their mutual interactions, a factor effect method is used (Jarošová \& Žaludová, 1997). The degree of influence of a factor on an observed variable is known as the factor effect, which is defined as the magnitude of response change caused by a change in the factor level. It is often referred to as the main factor effect, unlike the interaction effect.

If factor A' has two levels, then the main factor effect "A" is equal to the difference between the average response at one level and the average response at the second level (Jarošová \& Žaludová, 1997). If factor "A” has more than 2 levels, we usually use the effect of (i) level term of factor "A," which means the difference between the average response at level " $A_{i}$ " and the average of all the results from all the trials.

If the difference between response values at two levels of the factor " $A$ " are the same for different levels of factor "B," this implies that there is an interaction between factors from “A” and “B” (referred to as “AB”)(Jarošová \& Žaludová, 1997). 
The aim of this analysis is to determine the magnitude of each parameter's effect on the process. Proceeding based on the design matrix in Tab. 3.9, the parameters and interactions are assigned values of "+” and "-.” The next step is the sum of each of the examined parameter values (in our case, its density) with the aforementioned signage to a certain column, separated by a separator, which at experiment $2^{N}$ is generally $2^{N-1} r$. The following experiment matrix considers the effect of each of the examined factors $\left(\mathrm{p}, \mathrm{T}, \mathrm{w}_{\mathrm{r}}, \mathrm{L}\right)$, as well as their interactions $\left(\mathrm{pT}, \mathrm{pw}_{\mathrm{r}}, \mathrm{pL}, \mathrm{Tw}_{\mathrm{r}}, \mathrm{TL}, \mathrm{w}_{\mathrm{r}} \mathrm{L}, \mathrm{pTw} \mathrm{w}_{\mathrm{r}}, \mathrm{pTL}\right.$, $\mathrm{pw}_{\mathrm{r}} \mathrm{L}, \mathrm{Tw}_{\mathrm{r}} \mathrm{L}, \mathrm{p} \mathrm{Tw}_{\mathrm{r}} \mathrm{L}$ ). Factors with interactions are referred to according to the caption in Table 3.4, which is the established denotation.

According to the design matrix in Table 3.9, the magnitude of the main effects and their interaction are estimated. Estimated (calculated) values of each of the effects are shown in Table 3.9, and Fig. 3.14 illustrates the effect differences.

Table 3.8: The effect value of each parameter and their interaction.

\begin{tabular}{llllllll}
\hline B & C & BC & A & D & ABC & AC & AB \\
& 0.0333 & 0.0254 & 0.0181 & 0.012938 & 0.012881 & 0.012065 & 0.008587 \\
BCD & AD & ABCD & CD & ABD & ACD & BD & \\
\hline 0.00387 & 0.002897 & 0.002367 & 0.002005 & 0.000963 & 0.000661 & 0.000396 & \\
\hline
\end{tabular}

The magnitude of each effect expresses the weight of significance, magnitude of each parameter effect, and their interaction. It is obvious that the largest effects are the pressing temperature, material water content, and their interaction, followed by the pressing pressure and the fraction size, and finally by several mutual interactions between the examined parameters.

In relation to the proposed design, it is necessary to decide which of the listed examined parameters and interactions are to be considered in the mathematical model. 


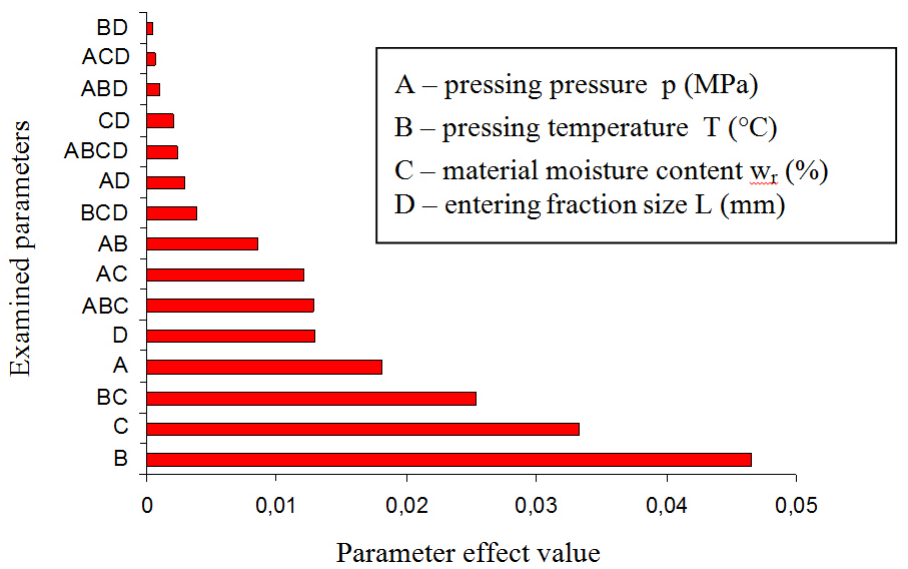

Figure 3.14: Paret's effect diagram.

Table 3.9: Main factors with interaction table-design matrix.

\begin{tabular}{|c|c|c|c|c|c|c|c|c|c|c|c|c|c|c|c|c|}
\hline $\begin{array}{l}\text { Number of } \\
\text { measurement }\end{array}$ & A & B & C & D & $A B$ & $A C$ & AD & BC & BD & CD & $A B C$ & ABD & ACD & BCD & $A B C D$ & $\rho_{\left(\mathrm{kg} \cdot \mathrm{dm}^{-3}\right)}$ \\
\hline 1 & -1 & -1 & -1 & -1 & 1 & 1 & 1 & 1 & 1 & 1 & -1 & -1 & -1 & -1 & 1 & 1.13919 \\
\hline 2 & 1 & -1 & -1 & -1 & -1 & -1 & -1 & 1 & 1 & 1 & 1 & 1 & 1 & -1 & -1 & 1.156558 \\
\hline 3 & -1 & 1 & -1 & 1 & -1 & 1 & -1 & -1 & 1 & -1 & 1 & -1 & 1 & -1 & 1 & 1.167468 \\
\hline 4 & 1 & 1 & -1 & 1 & 1 & -1 & 1 & -1 & 1 & -1 & -1 & 1 & -1 & -1 & -1 & 1.200171 \\
\hline 5 & -1 & -1 & 1 & -1 & 1 & -1 & 1 & -1 & 1 & -1 & 1 & -1 & 1 & 1 & -1 & 0.79969 \\
\hline 6 & 1 & -1 & 1 & -1 & -1 & 1 & -1 & -1 & 1 & -1 & -1 & 1 & -1 & 1 & 1 & 1.007282 \\
\hline 7 & -1 & 1 & 1 & 1 & -1 & -1 & -1 & 1 & 1 & 1 & -1 & -1 & -1 & 1 & -1 & 1.127726 \\
\hline 8 & 1 & 1 & 1 & 1 & 1 & 1 & 1 & 1 & 1 & 1 & 1 & 1 & 1 & 1 & 1 & 1.135111 \\
\hline 9 & -1 & -1 & -1 & 1 & 1 & 1 & -1 & 1 & -1 & -1 & -1 & 1 & 1 & 1 & -1 & 1.088649 \\
\hline 10 & 1 & -1 & -1 & 1 & -1 & -1 & 1 & 1 & -1 & -1 & 1 & -1 & -1 & 1 & 1 & 1.081203 \\
\hline 11 & -1 & 1 & -1 & -1 & -1 & 1 & 1 & -1 & -1 & 1 & 1 & 1 & -1 & 1 & -1 & 1.190808 \\
\hline 12 & 1 & 1 & -1 & -1 & 1 & -1 & -1 & -1 & -1 & 1 & -1 & -1 & 1 & 1 & 1 & 1.235596 \\
\hline 13 & -1 & -1 & 1 & 1 & 1 & -1 & -1 & -1 & -1 & 1 & 1 & 1 & -1 & -1 & 1 & 0.699423 \\
\hline 14 & 1 & -1 & 1 & 1 & -1 & 1 & 1 & -1 & -1 & 1 & -1 & -1 & 1 & -1 & -1 & 0.923658 \\
\hline 15 & -1 & 1 & 1 & -1 & -1 & -1 & 1 & 1 & -1 & -1 & -1 & 1 & 1 & -1 & 1 & 1.173846 \\
\hline 16 & 1 & 1 & 1 & -1 & 1 & 1 & -1 & 1 & -1 & -1 & 1 & -1 & -1 & -1 & -1 & 1.236333 \\
\hline
\end{tabular}




\subsection{Mathematical Model Design}

Based on the measured data obtained from experiment, we sought to find a relationship between a dependent variable, such as the briquette density $\rho$, and some relevant independent variables, such as the pressing pressure $p$, pressing temperature $T$, material moisture content $w_{r}$, and entering fraction size $L$.

We supposed that the dependency is given by a continuous function of four variables $\rho=f\left(p, T, w_{p} L\right)$, which is expanded into a Taylor's series. A mode, which includes linear variables and the interaction of independent linear variables, is assumed. The model can be simplified into a functional dependence:

$$
\rho=f(A, B, C, D, A B, A C, A D, B C, B D, C D, A B C, A B D, A C D, B C D, A B C D)
$$

Where:
A - Pressing pressure $\mathrm{p}(\mathrm{MPa})$
$\mathrm{B}$ - Pressing temperature $\mathrm{T}\left({ }^{\circ} \mathrm{C}\right)$
$\mathrm{C}$ - Material moisture content $\mathrm{w}_{\mathrm{r}}(\%)$
D - Entering fraction size $\mathrm{L}(\mathrm{mm})$

When the measured data represent a column vector of measured variable $\rho$ and a column vector of configured values of variables $A, B, C$, and $D$, then the dependency (3.7) can be described in the shape of a multiple linear regression model.

$$
\begin{aligned}
& \quad \rho_{i}=\beta_{0}+\beta_{1} \cdot A_{i}+\beta_{2} \cdot B_{i}+\beta_{3} \cdot C_{i}+\beta_{4} \cdot D_{i}+\beta_{5} \cdot A_{i} B_{i}+\beta_{6} \cdot A_{i} C_{i}+\beta_{7} \cdot A_{i} D_{i}+\beta_{8} \cdot B_{i} C_{i}+ \\
& +\beta_{9} \cdot B_{i} D_{i}+\beta_{10} C_{i} D_{i}+\beta_{11} A_{i} B_{i} C_{i}+\beta_{12} A_{i} B_{i} D_{i}+\beta_{13} A_{i} C_{i} D_{i}+\beta_{14} B_{i} C_{i} D_{i}+\beta_{15} A_{i} B_{i} C_{i} D_{i}+\varepsilon_{i} \\
& \text { for } i=1,2, \ldots . ., 16
\end{aligned}
$$

where:

$\beta_{j}$ - regressive parameters, for $j=0,1, \ldots ., 15$

$\varepsilon_{i}$ - measurement error

Then for estimating the coefficients of the regression function, the following applies:

$$
\hat{\beta}=\left(x^{T} x\right)^{-1} x^{T} \rho
$$

where:

$\hat{\beta}$ - variable parameter polynom estimation vector

$\mathrm{x}$ - plan matrix

$\rho$ - measured density value vector 
In this relation (3.9), the weight of each of the measurements is not taken into consideration. This is therefore necessary to expand the relation by a covariance matrix $U(\rho)$. The previous relation then becomes:

$$
\hat{\beta}=\left(x^{T} U^{-1}(\rho) x\right)^{-1} x^{T} U^{-1}\left({ }_{\rho}\right)_{\rho}
$$

Covariance matrix $U(\rho)$, where $m$ states the number of measurements, is as follows:

$$
U(\rho)=\left(\begin{array}{ccccccc}
u^{2}\left(\rho_{1}\right) & u\left(\rho_{1}, \rho_{2}\right) & u\left(\rho_{1}, \rho_{3}\right) & . & . & . & u\left(\rho_{1}, \rho_{m}\right) \\
u\left(\rho_{2}, \rho_{1}\right) & u^{2}\left(\rho_{2}\right) & u\left(\rho_{2}, \rho_{3}\right) & . & . & . & u\left(\rho_{2}, \rho_{m}\right) \\
\cdot & . & . & . & . & . & . \\
\cdot & . & . & . & \cdot & . & . \\
\cdot & \cdot & . & . & \cdot & . & u\left(\rho_{m-1}, \rho_{m}\right) \\
u\left(\rho_{m}, \rho_{1}\right) & u\left(\rho_{m}, \rho_{2}\right) & u\left(\rho_{m}, \rho_{3}\right) & . & . & u\left(\rho_{m}, \rho_{m-1}\right) & u^{2}\left(\rho_{m}\right)
\end{array}\right)
$$

For a closer description of designing the model from the measured data, it is necessary to decide which of the variables in the relation (3.7) should be included in the model and which can be disregarded.

\subsubsection{Regression Model Evaluation Criteria}

Some variables have a negligible effect on the density (Fig. 3.14) an can consequently be disregarded. A valid criterion is necessary to select use for the evaluation and comparison of variables in the model and regression model, respectively. Currently no theories exist that provide a precise specification of variables in a model. Nevertheless, there are several criteria that are suggested for use when selecting variable specifications for evaluating and comparing several regression models.

Initially, the criteria used are based on the coefficient of multiple determination $\underline{R}^{2}$ and corrected coefficient $R^{2}$, referred to as $R_{a d j}^{2}$. Coefficients are defined as follows (Palenčár \& et al., 2001):

$$
\begin{gathered}
R^{2}=\frac{S S_{A}}{S S_{T}}=1-\frac{S S_{R}}{S S_{T}} \\
R_{a d j}^{2}=1-\frac{S S_{R} /(n-k-1)}{S S_{T} /(n-1)}
\end{gathered}
$$


where:

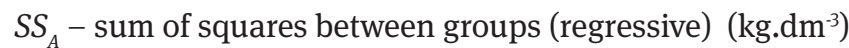

$S S_{R}$ - sum of squares inside the groups (residual) $\left(\mathrm{kg} \cdot \mathrm{dm}^{-3}\right)$

$S S_{T}$ - total sum of squares $\left(\mathrm{kg} \cdot \mathrm{dm}^{-3}\right)$

$(n-k-1)$ a $(n-1)$ - degrees of freedom

Coefficients of multiple determination $R^{2}$ and $R_{a d j}^{2}$ are based on the single factor variance analysis, which is a test of regression significance, or a test for determining if there is a linear relationship between variables $y$ and the subset of regressive variables (plan matrix columns) $x_{1}, x_{2}, \ldots . ., x_{k}$. Testing hypothesis $H_{0}: \mu_{1}=\mu_{2}=\ldots=\mu_{k}=0$, against the alternative $H_{1}$ : there results in at least one nonzero value of $\mu_{j}$. The rejection of $H_{0}: \mu_{1}=\mu_{2}=\ldots=\mu_{k}=0$ means that at least one of the regressive variables $x_{1}, x_{2}, \ldots$. $x_{k}$ contributes significantly to the model. Significance test of regression is a general procedure used in linear regression. The total sum of squares of the measured variable $S S_{T}$ is divided into 2 components:

$$
S S_{T}=S S_{A}+S S_{R}\left(\mathrm{~kg} \cdot \mathrm{dm}^{-3}\right)
$$

But first, it is necessary to calculate the sums of squares from the sum of values on the $\left(i^{\text {th }}\right)$ selection of $y_{i}$ and from the average values of this selection. The sum of squares between the groups is:

$$
S S_{A}=\sum_{i=1}^{k} \frac{y_{i}^{2}}{n_{i}}-\frac{1}{n} y^{2} . .\left(\mathrm{kg} . \mathrm{dm}^{-3}\right)
$$

Sum of squares inside the groups (residual) equals:

$$
S S_{R}=\sum_{i=1}^{k} \sum_{j=1}^{n_{i}} y_{i j}^{2}-\sum_{i=1}^{k} \frac{y_{i}^{2}}{n_{i}}\left(\mathrm{~kg} \cdot \mathrm{dm}^{-3}\right)
$$

The total sum of squares equals:

$$
S S_{T}=\sum_{i=1}^{k} \sum_{j=1}^{n_{i}} y_{i j}^{2}-\frac{1}{n} y^{2} .
$$

The results needed for testing $H_{0}$ are listed in Tab. 3.10. Hypothesis $H_{0}$ is reject on the level of significance $\alpha$ if $F>F(k-1, n-k, \alpha)$.

Other criteria also exist that can help in deciding whether including an additional explanatory variable would improve the model; in other words, other criteria can 
help determine which of the models we should prefer. One such criterion is known as Akaike's information criteria (AIC).

Table 3.10: Single factor variance analysis (Palenčár \& et al., 2001).

\begin{tabular}{lllll}
\hline $\begin{array}{l}\text { Variability } \\
\text { source }\end{array}$ & $\begin{array}{l}\text { Sum of squares } \\
\left(\mathrm{kg} . \mathrm{dm}^{-3}\right)\end{array}$ & $\begin{array}{l}\text { Degrees } \\
\text { of freedom }\end{array}$ & $\begin{array}{l}\text { Average square } \\
\left(\mathrm{kg}_{\mathrm{dm}} \mathrm{dm}^{-3}\right.\end{array}$ & Ration $\mathrm{F}$ \\
\hline Regressive sum & $\mathrm{SS}_{\mathrm{A}}$ & $\mathrm{k}-1$ & $M S_{A}=\frac{S S_{A}}{k-1}$ & $\mathrm{~F}=\mathrm{MS}_{\mathrm{A}} / \mathrm{MS}_{\mathrm{R}}$ \\
Residual sum & $\mathrm{SS}_{\mathrm{R}}$ & $\mathrm{n}-\mathrm{k}$ & $M S_{R}=\frac{S S_{R}}{n-k}$ & $\mathrm{~F}=\mathrm{MS}_{\mathrm{A}} / \mathrm{MS}_{\mathrm{R}}$ \\
Total sum & $\mathrm{SS}_{\mathrm{T}}$ & $\mathrm{n}-1$ & - & - \\
\hline
\end{tabular}

AIC is not a test of models on which basis we test the hypothesis; AIC is a tool for model selection (Akaike, 1981; Beck \& Arnold, 1977). The measured group of values or the designed models can be arranged and evaluated according to AIC with a distinction that the model with smallest AIC is the most appropriate. On the basis of AIC, it is possible to evaluate, for example, whether the best models are similar and whether they are much better than the rest of the models. It is not possible to set a value according to which a model is rejected using AIC.

Generally, AIC is calculated as follows (Akaike, 1981; Bozdogan, 1987):

$$
A I C=2 \cdot k_{m}-2 \cdot \ln \left(L_{p}\right)
$$

where:

$k_{m}$ - number of parameters in the model

$L_{p}-$ maximum value of the probable function for the estimated model

It is considered that model errors are normal and independently distributed. This method (AIC) tries to find a mode that best describes and explains the measured values and also includes at least 3 parameters. AIC decides how a model best describes the measured values. The AIC value only sorts and defines which model is best compared to the possible alternatives in the considered set of models.

AICc is AIC with a second degree of correction for samples with a small number of values and is calculated by (Akaike, 1981; Bozdogan, 1987):

$$
A I C c=A I C+\frac{2 k(k+1)}{n-k-1}
$$

Where: $n-k=$ degrees of freedom. 
The quality of estimated models is usually also measured by the RMSE criterion (Root mean squared error). RMSE is a very good test for model accuracy. The RMSE value is the root of the average sum of squares of the residuals (Palenčár \& et al., 2001). The average sum of squares of the residuals is the sum of squares divided by the difference between the number of observations and the number of estimated parameters. Then, the best model from the designed alternatives is the model that has the smallest standard deviation of the residuals.

Based on the three previously described criteria, we will compare and evaluate the designed models with the variables.

\subsubsection{Regression Model Creation and Selection}

This section will focus on the creation and selection of an appropriate regression model based on the criterion mentioned in previous chapter. For the selection and evaluation of models, the software JMP 8 is used. This software calculates the values for each of the described criteria for each designed mode. Based on the results obtained from the factor effect method selected, the parameter effect values in Model 1 and Models 2 and 3, respectively, are shown in Fig. 3.15..

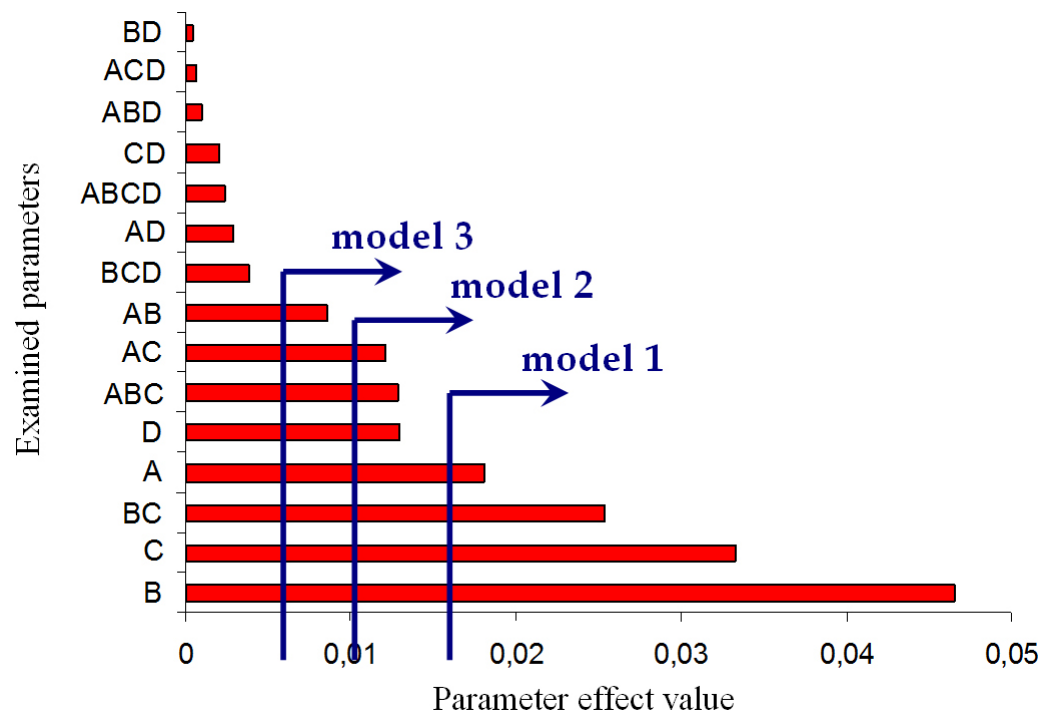

Figure 3.15: Variable and regression model design selection example. 
With the aid of JMP 8, we have calculated the values for the observed criteria (Tab. 3.11). Model 1, which considers 4 factors and is based on measurement results having the largest effect, is described by the list of values of measured data with $87.9 \%$ accuracy. This accuracy cannot be disregarded, but to improve accuracy, more parameters were added to the model (Model 2). The model's accuracy was indeed increased with the addition of more parameters. Additionally, the criterion RMSE was implies that Model 2 is better than Model 1. However, the AICc criterion implies that Model 2 is worse compared to Model 1. An additional parameter (Model 3) was thus added, and this designed model was evaluated by all three criteria. These three criteria indicate that Model 3 is the most accurate..

Subsequently, this simplified approach for design was also applied to select a regression model that considers all the variables according to Equation 3.7. The Software JMP 8 created models from all possible combinations of variables from Equation 3.7 and calculated the values of the examined criteria for each mode. In Tab 3.11, the models and their coefficients are listed. Appropriate models were selected based on the highest values of the coefficient $R^{2}$, the lowest values of $A I C c$, and the lowest values of the coefficients for RMSE.

Table 3.11: The best models calculated with JMP 8on the basis of the evaluating criterion.

\begin{tabular}{llllll}
\hline Model & Parameters & $\begin{array}{l}\text { Number of } \\
\text { parameters }\end{array}$ & $\mathbf{R}^{2}$ & AICC & RMSE \\
\hline model 1 & B,C,BC,A & 4 & $87.9 \%$ & -30.1238 & 0.058448 \\
model 2 & B,C,BC,A,D,ABC,AC & 7 & $96.8 \%$ & -24.8346 & 0.035139 \\
model 3 & B,C,BC,A,D,ABC,AC,AB & $\mathbf{8}$ & $99.3 \%$ & -32.7996 & $\mathbf{0} .017764$ \\
model 4 & C,AB,AC,BC,ABCD & 5 & $94.3 \%$ & -35.5702 & 0.041982 \\
model 5 & B,C,AB,AC,BC, ABCD & 6 & $96.1 \%$ & -33.239 & 0.036413 \\
model 6 & B, C, BC, A, D & 5 & $91.3 \%$ & -28.8272 & 0.05183 \\
\hline
\end{tabular}

All of the mentioned models are solved with a certain accuracy describing the measured data. Each of the mentioned models could be used depending on specific conditions described by the models. For selecting a model, which will be discussed later, it is necessary to define the initial conditions; if the simplest model from a statistical point of view is desirable, the model with the least number of parameters would be chosen (Model 1).

Since the model is designed for the densification process (pressing), the model must include all of the examined parameters (A, B, C, D) because the final model must describe the effects of all four examined parameters on the resultant density of the 
briquette. In terms of the densification process, the models should also include the mutual interaction of the parameters, which have a significant effect on the resultant density of the briquettes. Selecting an appropriate model requires an objective evaluation and comparison of each of the values of the examined criterion for each group of models. That is why that, from the six models, Model 3 is ultimately chosen. Model 3 describes the list of measured data with $99.3 \%$ accuracy, and the value of the RMSE coefficient is the lowest. The AICc criterion is not the lowest for this model, but considering the values of the coefficients $R^{2}$ and RMSE, it is still the best choice. Model 3 includes all four examined parameters (A, B, C, D) and their interactions, which have a significant effect on the density of the briquette (BC). This model also includes the interaction of some important parameters (ABC, $\mathrm{AC}$, and $\mathrm{AB}$ ). Based on what has previously been discussed, the dependency in Equation 3.7 can now be rewritten to yield the following form:

$$
\rho=f(A, B, C, D, A B, A C, B C, A B C)
$$

Equation 3.8 can also be rewritten to have a multiple linear regression model form:

$$
\begin{gathered}
\rho_{i}=\beta_{0}+\beta_{1} \cdot A_{i}+\beta_{2} \cdot B_{i}+\beta_{3} \cdot C_{i}+\beta_{4} \cdot D_{i}+ \\
+\beta_{5} \cdot A_{i} B_{i}+\beta_{6} \cdot A_{i} C_{i}+\beta_{7} \cdot B_{i} C_{i}+\beta_{8} \cdot A_{i} B_{i} C_{i}+\varepsilon_{i}
\end{gathered}
$$

for $i=1,2, \ldots . ., 16$

where:

$\beta_{j}$ - are regressive parameters, for $j=0,1, \ldots . ., 8$

$\varepsilon_{i}$ - measurement error

\subsubsection{Mathematical Model Design}

This section will deal with the calculation or estimation of regressive parameters $\beta_{j}$, for $j=0,1, \ldots . . ., 8$. Once again, the JMP 8 software was employed, with which the values of the regressive parameters $\beta_{j}$ are calculated for each regression model. For Model 3, and according to Equation 3.21, the regressive parameters have estimated values shown in Tab. 3.12. In the left column, there are variables that contain the designed and selected model. In the second column from the left, regressive parameters attributed to variables in the first column are listed. In the third column from the left, specific estimated values of the regressive parameters are shown. Also here we see the standard estimated values of the regressive parameters and the values of the upper and lower limit of 95\% the reliability interval. Considering the values of the regressive parameters, we can rewrite Equation 3.21 into the form 3.22. 
Table 3.12: Estimated regressive parameters values using JMP 8 software.

\begin{tabular}{|c|c|c|c|c|c|}
\hline $\begin{array}{l}\text { Model } \\
\text { variables }\end{array}$ & $\begin{array}{l}\text { Regressive } \\
\text { parameters }\end{array}$ & $\begin{array}{l}\text { Estimated } \\
\text { value }\end{array}$ & $\begin{array}{l}\text { Standard } \\
\text { error }\end{array}$ & $\begin{array}{l}\text { Lower limit } \\
\mathbf{9 5 , 0 \% \text { interval }}\end{array}$ & $\begin{array}{l}\text { Upper limit } \\
\mathbf{9 5 , 0 \%} \text { interval }\end{array}$ \\
\hline & $\beta_{0}$ & 4.98371 & 0.236318 & 4.52053 & 5.44688 \\
\hline A & $\beta_{1}$ & -0.0261781 & 0.00189126 & -0.029885 & -0.0224713 \\
\hline B & $\beta_{2}$ & -0.0410292 & 0.00228295 & -0.0455038 & -0.0365547 \\
\hline C & $\beta_{3}$ & -0.620594 & 0.0235536 & -0.666758 & -0.574429 \\
\hline D & $\beta_{4}$ & -0.015446 & 0.00152757 & -0.01844 & -0.012452 \\
\hline$A B$ & $\beta_{5}$ & 0.000228845 & 0.0000185268 & 0.000192533 & 0.000265157 \\
\hline$A C$ & $\beta_{6}$ & 0.0031851 & 0.000188348 & 0.00281595 & 0.00355426 \\
\hline BC & $\beta_{7}$ & 0.00528717 & 0.000230218 & 0.00483595 & 0.00573839 \\
\hline$A B C$ & $\beta_{8}$ & -0.0000273004 & 0.00000185582 & -0.0000309378 & -0.000023663 \\
\hline sigma & sigma & 0.00586314 & 0.00110664 & 0.00405013 & 0.00848773 \\
\hline
\end{tabular}

$$
\begin{gathered}
\rho_{i}=4,98371-0,0261781 . A_{i}-0,0410292 . B_{i}-0,620594 . C_{i}- \\
-0,015446 \cdot D_{i}+0,000228845 . A_{i} B_{i}+ \\
+0,0031851 . A_{i} C_{i}+0,00528717 . B_{i} C_{i}-0,0000273004 . A_{i} B_{i} C_{i}+\varepsilon_{i}\left(\mathrm{~kg} . \mathrm{dm}^{-3}\right)
\end{gathered}
$$

for $i=1,2, \ldots . ., 16$

This equation is not the final mode; it is a multiple linear regression model with estimated values of regressive parameters. It is necessary to further design the mathematical model. For calculating and designing a mathematical model, JMP 8 software is once again utilized, which designed the model in the following form:

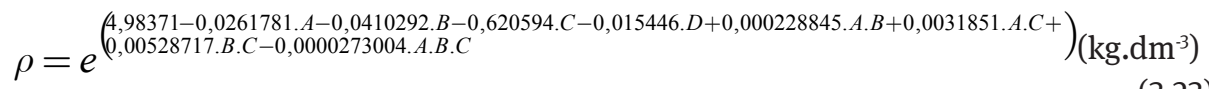

Substituting the notation of the variables or the examined parameters we can rewrite the relation to:

$\rho=e^{\left(\begin{array}{l}4,98371-0,0261781 \cdot p-0,0410292 \cdot T-0,620594 \cdot w_{r}-0,015446 \cdot L+0,000228845 \cdot p \cdot T+ \\ 0,0031851 \cdot p \cdot w_{r}+0,00528717 \cdot T \cdot w_{r}-0,0000273004 \cdot p \cdot T \cdot w_{r}\end{array}\right)}\left(\mathrm{kg} \cdot \mathrm{dm}^{-3}\right)$

Equation 3.24 can be rewritten into the following form: 


$$
\begin{array}{r}
\ln \rho=4,98371-0,0261781 \cdot p-0,0410292 \cdot T-0,620594 \cdot w_{r}-0,015446 \cdot L+0,000228845 . p \cdot T \\
+0,0031851 . p \cdot w_{r}+0,00528717 \cdot T \cdot w_{r}-0,0000273004 . p \cdot T \cdot w_{r}
\end{array}
$$

Then, using the designed mathematical model (according to 3.24), the density values at levels according to the experimental plan in (Tab. 3.13) are calculated and compared with the densities of the briquettes obtained from experiments at these configurations.

The following graph (Fig. 3.16) illustrates the comparison of the experimentally measured density values with the calculated density values. In this graph, it can be seen that the characters for both are almost identical; this comparison proves that the proposed procedure was appropriate when selecting a regression model based on the criteria $R^{2}, A I C c$, and RMSE.

Table 3.13: Comparing briquette densities obtained from the experiments and calculated with model.

\begin{tabular}{lllllll}
\hline n.m. & $\mathbf{p}(\mathrm{MPa})$ & $\mathbf{T}\left({ }^{\circ} \mathrm{C}\right)$ & $\mathbf{w}(\%)$ & $\mathbf{L}(\mathbf{m m})$ & $\begin{array}{l}\boldsymbol{\rho}_{\mathbf{n}} \text { experiment } \\
\left(\mathbf{k g . d m} \mathbf{d m}^{-3}\right.\end{array}$ & $\begin{array}{l}\boldsymbol{\rho}_{\mathrm{n}} \text { from model } \\
\left(\mathbf{k g . d m} \mathbf{m}^{-3}\right)\end{array}$ \\
\hline 1 & 95 & 85 & 8 & 1 & 1.139 & 1.138 \\
2 & 159 & 85 & 8 & 1 & 1.157 & 1.152 \\
3 & 95 & 115 & 8 & 4 & 1.167 & 1.163 \\
4 & 159 & 115 & 8 & 4 & 1.200 & 1.201 \\
5 & 95 & 85 & 12 & 1 & 0.799 & 0.797 \\
6 & 159 & 85 & 12 & 1 & 1.007 & 1.007 \\
7 & 95 & 115 & 12 & 4 & 1.128 & 1.125 \\
8 & 159 & 115 & 12 & 4 & 1.135 & 1.176 \\
9 & 95 & 85 & 8 & 4 & 1.089 & 1.087 \\
10 & 159 & 85 & 8 & 4 & 1.081 & 1.100 \\
11 & 95 & 115 & 8 & 1 & 1.191 & 1.218 \\
12 & 159 & 115 & 8 & 1 & 1.236 & 1.258 \\
13 & 95 & 85 & 12 & 4 & 0.699 & 0.761 \\
14 & 159 & 85 & 12 & 4 & 0.924 & 0.961 \\
15 & 95 & 115 & 12 & 1 & 1.174 & 1.178 \\
16 & 159 & 115 & 12 & 1 & 1.236 & 1.231 \\
\hline
\end{tabular}




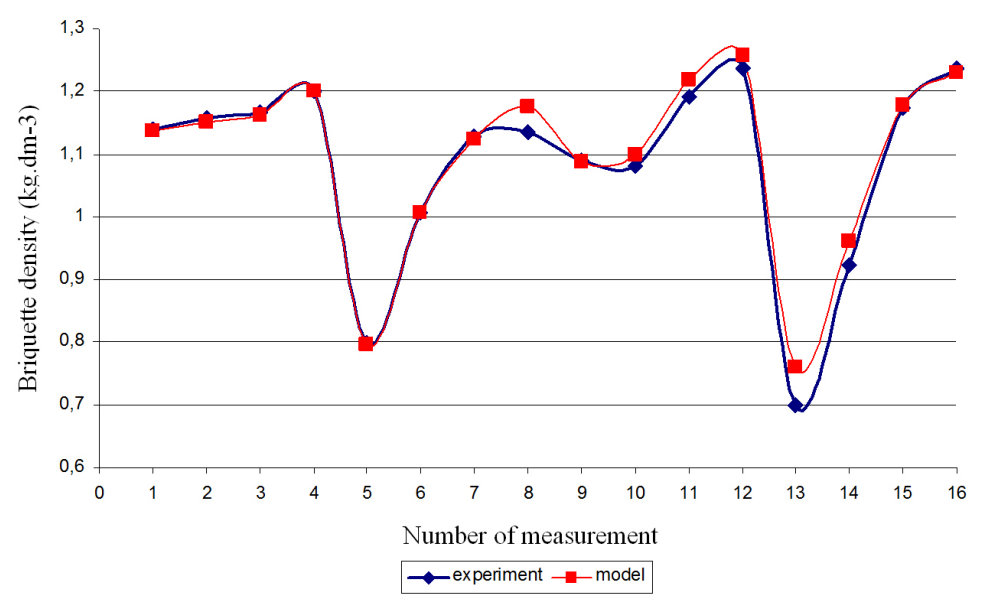

Figure 3.16: Graphic comparison of briquette densities obtained from experiments and calculated from the regression model.

\subsubsection{Measurement Errors}

Measurement error is a parameter related to the results of the measurement, which characterises the variance of measured values. Error is included in the measurement results, the values taken by the used instrument, the values of used constants, and so on. Each effect registered in the measurement process or somehow affecting the process brings a certain level of inaccuracy to the measurement itself. These inaccuracies cause errors in the resultant density of the briquette. We can say that the error of the measured result reflects defects in the perfect knowledge of the density value. Phenomena that contribute to error so that the results cannot be characterized by one value are called error sources.

The basic quantitative error characteristic is the standard error $u$. Standard error is equal to the standard deviation of the respective probability distribution as it is considered that the measured values and errors have a probability distribution (Chudý \& et al., 1999).

Errors from each source can be evaluated using two basic methods (Chudý \& et al., 1999):

a) Errors from statistical methods from measured data are known as Error defined by method A (Errors type A) - $u_{A}$;

b) Errors from any other source, defined as errors by method B (Errors type B) $-u_{B}$.

Contrary to errors evaluated by method A, where the causes of the errors are generally considered as unknown, the errors evaluated by method B are from known or 
unidentifiable sources. A characteristic sign of the errors evaluated by method A is that with more measurements, their values decrease. Errors evaluated by method B are independent to the number of measurements. It is necessary to remember that the errors evaluated by both methods are equivalent and differ only in the manner of evaluation; however, in terms of magnitude, they differ and some can be neglected.

Combining the standard error evaluated by method A and standard error evaluated by method B yields the total (combined) standard error $u_{c}$ (Chudý \& et al., 1999). Standard errors constitute an interval covering the real value of the measured variable with a relatively small probability (around 66\%). Usually, we require a value of error that creates an interval with a larger probability of covering the real value. That is why the expanded error $U$ is used:

$$
U=k \cdot u_{C}
$$

\section{Procedure for evaluating type A}

Evaluation type A of standard error, as has been mentioned before, is a method of evaluation with the aid of statistical analysis of the measured data. The statistical evaluation depends on the model of measurement. In the case of repeated direct measurements, it is about the statistical processing of values of repeated direct measurements. Most frequently, we consider that the measured values are obtained with independent measurements under the same conditions. This means that, if we have $n$ measured values $x_{1}, x_{2}, \ldots, x_{n}$, the measured values are the realization of $n$ independent equally precise measurements of one variable. Then the following is true:

- estimated values of measured variables according to the relation:

$$
\bar{x}=\frac{1}{n} \sum_{i=1}^{n} x_{i}
$$

- standard error is determined by method A; this estimation is equal to the standard variation of the arithmetic average. Thus:

$$
u(x)=s(\bar{x})=\frac{s(x)}{\sqrt{n}}=\sqrt{\frac{1}{n(n-1)} \sum_{i=1}^{n}\left(x_{i}-\bar{x}\right)^{2}}
$$

Procedure for evaluating type B

As was mentioned before, the evaluation of error using method B is everything but the statistical analysis of measured values. Amongst the most frequent way of evaluating by type B are: 
1. If the certificates, documentation from the manufactures or other sources, list the expanded error $U$ and the expansion coefficient $k$, then method B for evaluating the standard error $u\left(z_{i}\right)$ from the given source $Z_{i}$ lies in the equation:

$$
u\left(z_{i}\right)=\frac{U}{k}
$$

2. If $2 U$ is the length of the interval with a confidential probability (95\%, $99 \%$, $99.73 \%$ ), and it can be said that, when determining this interval, the normalized normal distribution was considered, standard error $\left(z_{i}\right)$ from the source $Z_{i}$ is determined form the following equation:

$$
u\left(z_{i}\right)=\frac{U}{k_{p}}
$$

where $k_{p}$ is the expansion coefficient, which equals the quantile of normalized normal distribution for a probability of $P_{p}\left(k_{p}=1,96\right.$ pre $P_{p}=95 \%$; $k_{p}=2,58$ pre $P_{p}=99 \%$; $k_{p}=3$ for $\left.P_{p}=99.73 \%\right)$.

3. It is possible to estimate only the boundaries in which the values of the variable are located under the effect of the given source at almost 100 percent. For this estimation, we proceed as follows:

- estimate the rate of change (error) $\pm z_{\text {imax }}$ of the nominal value of the variable related to the considered source $Z_{i}$, which is very unlikely (practically impossible);

- the probability distribution of the error is evaluated in this interval, and its approximation is determined;

- standard error of the considered source $u\left(z_{i}\right)$ is calculated from the relation:

$$
u\left(z_{i}\right)=\frac{z_{i \max }}{k}
$$

where $k$ is the value related to the selected approximation of the probability distribution:

- for normal distribution (N) $k=3(k=2)$;

- for equal (right-angled) distribution (R);

- for triangular distribution (S) $k=2,45$;

- $\quad$ for bimodal distribution (U) $k=1$. 
The approximation with normal distribution is used if deviations from nominal values of small values may occur more often, and the resulting increase in probability decreases value.

The approximation with equal distribution is used if the probability of occurrence of any error in the interval is the same $\pm z_{\text {imax }}$. This approximation is the most frequently used because it common to not have enough knowledge about the probability of error occurrence distribution, and thus some of the errors cannot be biased. This distribution is considered in this work.

Triangular distribution approximation is used just like the normal one.

Bimodular distribution approximation can be used, for example, for measurement instruments, which the manufacturer sorts into several accuracy classes (classification of measurement instruments).

The aforementioned methods of evaluation (Type B) of the standard error of course do not constitute all possibilities. Measurements in practice can vary, and it is very important for each situation to closely consider all circumstances that can act as an error source.

While using a numerical measurement instrument, the source of error is the last valid resolution number. This means that, despite the fixed nature of the value after repeated measurements, the error is not zero. Error estimation in this case is based on the consideration of equal probability distribution in the interval restricted by the resolution $\delta\left(z_{i}\right)$ of the given instrument. In the case of analog measurement instruments, the readability is usually given by the value of the segments scale. Usually, it has also a significant effect on the instrument hysteresis.

\section{Determining standard errors when measuring}

We considering the case when the value of $Y$ (output value), which interests us, is a function $f$ of values $X_{1}, X_{2}, \ldots X_{m}$. Values $X_{1}, X_{2}, \ldots X_{m}$ (input values) represent a directly measured value (affecting values, corrections, physical constants, etc.), so values that can be directly measured or have known estimations, errors, and covariances from other sources. We can write:

$$
Y=f\left(X_{1}, X_{2}, \ldots, X_{m}\right)
$$

where $f$ is a known function.

Estimate $y$ of output value $Y$ is determined from the equation:

$$
y=f\left(x_{1}, x_{2}, \ldots, x_{m}\right)
$$

where $x_{1}, x_{2}, \ldots, x_{m}$ are the estimates of the input values $X_{1}, X_{2}, \ldots, X_{m}$. 
Estimate $y$ of value $Y$ error indicates that the estimates $x_{1}, x_{2}, \ldots, x_{m}$ are uncorrelated. From the relation (if we linearize the function $f$ by expanding it into a Taylor's series and neglect higher terms) we get:

$$
u^{2}(y)=\sum_{i=1}^{m} A_{i}^{2} u^{2}\left(x_{i}\right)
$$

Where, for coefficients (sensitivity, conversion) $A_{i}$, it is true that:

$$
A_{i}=\left|\frac{\partial f\left(X_{1}, X_{2}, \ldots, X_{m}\right)}{\partial X_{i}}\right|_{X_{1}=x_{1}, \ldots \ldots, X_{m}=x_{m}}
$$

The designed mathematical model in this manuscript (Equation 3.24) can be written in its basic form as $\rho=f\left(p, T, w_{r}, L, p T, p w_{r}, T w_{p}, p T w_{r}\right)$, where $\rho$ is the density of the briquette, and the values for $p, T, w_{p} L$ and their interactions are configured and therefore known to affect the resultant observed value of $\rho$. The observed value $\rho$ is calculated according to a known equation for density (2.13) and an equation for calculating the cylinder volume. Based on what has been mentioned, and after adapting the relations to our case, we are able to rewrite relation (3.32) into the following form

$$
\rho=\frac{m_{n}}{V_{n}}=\frac{m_{n}}{\pi \cdot \frac{d_{n}^{2}}{4} \cdot L_{n}}
$$

Throughout the experiments, after pressing, values $m_{n}, d_{n}$, and $L_{n}$, are measurable, which means that we make an indirect measurement of one value. The measurements do not consider covariances as the measured value estimates are uncorrelated because the values of $m_{n}, d_{n}$, and $L_{n}$ are measured with a different instrument. With these considerations, Equation 3.34 and Equation 3.35 can be written for this case as follows:

$$
u \rho=\sqrt{\left(\frac{\partial \rho}{\partial m_{n}}\right)^{2} \cdot u_{m n}^{2}+\left(\frac{\partial \rho}{\partial d_{n}}\right)^{2} \cdot u_{d n}^{2}+\left(\frac{\partial \rho}{\partial L_{n}}\right)^{2} \cdot u_{L n}^{2}}
$$

where:

$u_{m n}$ is the total combined standard error of the instrument (scale) for measuring the value $m_{n}$ (weight of briquette) 
$u_{d n}$ is the total combined standard error of the instrument (calliper 1) for determining the value $d_{n}$ (briquette diameter);

$u_{L n}$ is the total combined standard error of the instrument for measuring the value of the mold $L_{n}$ (briquette length);

Sometimes, it is advantageous to determine the errors of estimates $y$ of output value $Y$ by methods A and method B separately. Then, the total (combined) standard error will be given with the relation (if we do not consider the dependency between the sources of error evaluated by method type A and method type B):

$$
u(y)=\sqrt{u_{A}^{2}(y)+u_{B}^{2}(y)}
$$

Based on Equation 3.38 for this case, it is true that:

$$
\begin{aligned}
& u_{m n}^{2}=u_{A m n}^{2}+u_{B m n}^{2} \\
& u_{d n}^{2}=u_{A d n}^{2}+u_{B d n}^{2} \\
& u_{L n}^{2}=u_{A L n}^{2}+u_{B L n}^{2}
\end{aligned}
$$

Since there were 7 measurements at 16 different configurations, Equation 3.27 can be written as follows:

$$
\begin{aligned}
& \bar{m}_{n}=\frac{1}{7} \sum_{i=1}^{7} m_{n i} \\
& \bar{d}_{n}=\frac{1}{7} \sum_{i=1}^{7} d_{n i} \\
& \bar{L}_{n}=\frac{1}{7} \sum_{i=1}^{7} L_{n i}
\end{aligned}
$$

Then, considering relations 3.42-3.44, we can then rewrite the standard errors determined by method A based on Equation 3.28 and calculate it according to the following equations: 


$$
\begin{aligned}
& u_{A m n}=\sqrt{\frac{1}{7(7-1)} \sum_{i=1}^{7}\left(m_{n i}-\bar{m}_{n}\right)^{2}} \\
& u_{A d n}=\sqrt{\frac{1}{7(7-1)} \sum_{i=1}^{7}\left(d_{n i}-\bar{d}_{n}\right)^{2}} \\
& u_{A L n}=\sqrt{\frac{1}{7(7-1)} \sum_{i=1}^{7}\left(L_{n i}-\overline{L_{n}}\right)^{2}}
\end{aligned}
$$

For the standard error determined by method B, it is true that:

$$
u_{B}^{2}(y)=u_{B 1}^{2}(y)+u_{B 2}^{2}(y)+\ldots .
$$

where:

- $u_{B 1}^{2}(y)$ is the instrument error, whose value is according to a certification less than or equal to $\delta_{d o v}$. Assuming equal distribution to be

$u_{B 1}^{2}(y)=\frac{\delta_{d o v}}{\sqrt{3}}$, according to Equation 3.31.

- $u_{B 2}^{2}(y)$ is the error of readability and so on, but this error is neglected.

We determine the standard error type B from the following Equations 3.49-3.51. The values $\delta_{d o v}$ of each instrument was listed by the manufacturer in the attached certificates. Then, the standard error type B will have values as follows:

$$
\begin{aligned}
& u_{B m n}^{2}=\frac{\delta_{d o v} v}{\sqrt{3}}=\frac{0,02}{\sqrt{3}}=0,00013333 \\
& u_{B d n}^{2}=\frac{\delta_{d o v} p_{1}}{\sqrt{3}}=\frac{0,025}{\sqrt{3}}=0,00020832 \\
& u_{B L n}^{2}=\frac{\delta_{d o v} p_{2}}{\sqrt{3}}=\frac{0,02}{\sqrt{3}}=0,00013333
\end{aligned}
$$

Sensitivity coefficients coming from relation 3.37 are used to calculate according to relation 3.35, and they will have the following form:

$$
A_{m n}=\frac{\partial \rho}{\partial m_{n}}=\frac{4}{\pi \cdot d_{n}{ }^{2} \cdot L_{n}}
$$




$$
\begin{aligned}
& A_{d n}=\frac{\partial \rho}{\partial d_{n}}=-\frac{8 \cdot m_{n}}{\pi \cdot d_{n}^{3} \cdot L_{n}} \\
& A_{L n}=\frac{\partial \rho}{\partial L_{n}}=-\frac{4 \cdot m_{n}}{\pi \cdot d_{n}^{2} \cdot L_{n}^{2}}
\end{aligned}
$$

On the basis of previous Equations 3.42-3.54, all of the coefficients and values needed for expressing the standard error type A and B are calculated according to Equations 3.39-3.41. Subsequently, the errors of estimation of the observed values $\rho_{\mathrm{n}}$ at each of the 16 configurations according to Equation 3.37 are calculated. The calculated errors are listed below in the table of errors.

Table 3.14: Table of calculated measurement errors for each configuration.

\begin{tabular}{lll}
\hline $\mathrm{n} . \mathrm{m}$. & $\boldsymbol{\rho}_{\mathrm{n}}\left(\mathrm{kg}_{\mathrm{dm}}{ }^{-3}\right)$ & $\mathrm{u}_{\mathrm{p}}\left(\mathrm{kg}^{\mathrm{d}} \mathrm{dm}^{-3}\right) .10^{-3}$ \\
\hline 1 & 1.134889752 & 0.0482210 \\
2 & 1.156558042 & 0.0562181 \\
3 & 1.16746774 & 0.186039 \\
4 & 1.205601254 & 0.0466928 \\
5 & 0.799690152 & 0.306733 \\
6 & 1.007281758 & 0.184125 \\
7 & 1.127725754 & 0.0832159 \\
8 & 1.135110519 & 0.12598 \\
9 & 1.088648941 & 0.156747 \\
10 & 1.081203369 & 0.187254 \\
11 & 1.190807876 & 0.187356 \\
12 & 1.235596 & 0.212271 \\
13 & 0.754723313 & 0.103315 \\
14 & 0.960492782 & 0.201452 \\
15 & 1.173846102 & 0.132002 \\
16 & 1.236332914 & 0.197956 \\
\hline
\end{tabular}

